\title{
Functional central limit theorems for stick-breaking priors
}

\author{
Yaozhong $\mathrm{Hu}^{*, \dagger}$, Junxi Zhang*
}

\section{Supplemental Appendix}

In this section, we present the necessary definitions and propositions for the processes (random measures) considered in this paper and give the proofs for the propositions and theorems in the main body.

\subsection{Definitions}

Let $(\Omega, \mathcal{F}, \mathbb{P})$ be a complete probability space and let $(\mathbb{X}, \mathcal{X})$ be a measurable Polish space, namely, $\mathbb{X}$ is a separable complete metric space and $\mathcal{X}$ is the Borel $\sigma$-algebra of $\mathbb{X}$. Let $H$ be a nonatomic probability measure on $(\mathbb{X}, \mathcal{X}$ ) (i.e. $H(\{x\})=0$ for any $x \in \mathbb{X}$ ). A random measure is a mapping $P$ from $\Omega \times \mathcal{X}$ to $\mathbb{R}_{+}$(we denote this random measure by $P=(P(\omega, A), \omega \in \Omega, A \in \mathcal{X}))$ such that

(i) when $\omega \in \Omega$ is fixed, $P(\omega, \cdot)$ is a measure on $(\mathbb{X}, \mathcal{X})$;

(ii) when $A \in \mathcal{X}$ is fixed, $P(\cdot, A)$ is a random variable on $(\Omega, \mathcal{F}, \mathbb{P})$.

In the following definitions we shall always assume that $P$ is a random probability measure, which are given by their stick-breaking representation. Different assumptions on the stick-breaking weights give rise to different processes. The first one is the classical Dirichlet process.

Definition 7.1. Let $a>0$ and let $H$ be a nonatomic measure on $(\mathbb{X}, \mathcal{X})$. A random probability measure $P$ is called the Dirichlet process with parameter $(a, H)$, denoted by $P \sim \operatorname{DP}(a, H)$, if it has the representation $(2.1)-(2.2)$, where $v_{i} \stackrel{i i d}{\sim} \operatorname{Beta}(1, a)$.

In fact, this process is defined differently and the above definition is a result of Sethuraman (1994). To state the original definition of the Dirichlet process as the following proposition, we need to recall the concept of the Dirichlet distribution. Throughout this paper we use the following notation to denote the standard simplex in $\mathbb{R}^{n}$ :

$$
\mathbb{S}_{n}=\left\{\left(s_{1}, \cdots, s_{n}\right) \in \mathbb{R}^{n}: \quad s_{i} \geq 0, \quad \sum_{i=1}^{n} s_{i}=1\right\} .
$$

\footnotetext{
*Department of Mathematical and Statistical Sciences, University of Alberta at Edmonton, Alberta, Canada, T6G2G1 yaozhong@ualberta.ca junxi3@ualberta.ca

${ }^{\dagger}$ Supported by an NSERC discovery fund and a startup fund of University of Alberta.

(C) 2021 International Society for Bayesian Analysis
} 
In case of no ambiguity we also write $\mathbb{S}=\mathbb{S}_{n}$. A random vector $\left(X_{1}, \cdots, X_{n}\right) \in$ $\mathbb{S}$ follows the Dirichlet distribution with parameters $\left(\alpha_{1}, \cdots, \alpha_{n}\right) \in[0, \infty)^{n}$, denoted by $\left(X_{1}, \cdots, X_{n}\right) \sim \operatorname{Dir}\left(\alpha_{1}, \cdots, \alpha_{n}\right)$, if the joint probability density function of $\left(X_{1}, \cdots, X_{n}\right)$ is given by

$$
f\left(x_{1}, \cdots, x_{n}\right)=\frac{\Gamma(|\alpha|)}{\prod_{i=1}^{n} \Gamma\left(\alpha_{i}\right)} \prod_{i=1}^{n} x_{i}^{\alpha_{i}-1} \mathbb{1}_{\mathbb{S}}\left(x_{1}, \cdots, x_{n}\right),
$$

where $|\alpha|=\sum_{i=1}^{n} \alpha_{i}, \Gamma(a)=\int_{0}^{\infty} x^{a-1} d x(a>0)$, is the gamma function, and $\mathbb{1}_{\mathbb{S}}$ is the indicator function of the simplex $\mathbb{S}$. With this notion of Dirichlet distribution we can write the following proposition.

Proposition 7.2. A random probability measure $P$ is the Dirichlet process with parameter $(a, H)$ if for any measurable partition $\left(A_{1}, \cdots, A_{n}\right)$ of $\mathbb{X}$ (i.e. $A_{1}, \cdots, A_{n} \in \mathcal{X}, A_{1} \cup$ $\cdots \cup A_{d}=\mathbb{X}$ and $A_{i} \cap A_{j}=\emptyset$ for $\left.1 \leq i<j \leq n\right)$, the random vector $\left(P\left(A_{1}\right), \cdots, P\left(A_{n}\right)\right)$ follows the Dirichlet distribution with parameters $\left(a H\left(A_{1}\right), \cdots, a H\left(A_{n}\right)\right)$.

Proof. We refer to Sethuraman (1994) or Hu and Zhang (2020) for the proof of the equivalence between Definition 7.1 and Proposition 7.2.

Definition 7.3 (Pitman and Yor (1997)). Let $b \in(0,1)$ and let $-b<a<\infty$. A random probability measure $P$ is called the two-parameter Poisson-Dirichlet process or the Pitman-Yor process, denoted by $\operatorname{PDP}(a, b, H)$, if the stick-breaking weights satisfy the following:

$$
\left\{\begin{array}{l}
v_{1}, v_{2}, \cdots \text { are independent, } \\
v_{i} \sim \operatorname{Beta}(1-b, a+i b), \quad i=1,2, \cdots
\end{array}\right.
$$

Definition 7.4 (Favaro et al. (2012)). A random probability measure $P$ is called the normalized inverse Gaussian process with parameters a and $H$, denoted by $P \sim \mathrm{N}-\mathrm{IG}(a, H)$, if the joint distributions of the stick-breaking weights $\left\{v_{1}, v_{2}, \cdots\right\}$ are given through the following conditional probability densities recursively:

$$
\left\{\begin{array}{c}
f_{v_{1}}(x)=\frac{a^{\frac{1}{2}} x^{-\frac{1}{2}}(1-x)^{-1}}{(2 \pi)^{\frac{1}{2}} K_{-\frac{1}{2}}(a)} K_{-1}\left(\frac{a}{\sqrt{1-x}}\right), \\
f_{v_{n} \mid v_{1}, \cdots, v_{n-1}}(x)=\frac{a^{\frac{1}{2}} \prod_{i=1}^{n-1}\left(1-v_{i}\right)^{-\frac{1}{4}} x^{-\frac{1}{2}}(1-x)^{-\frac{5}{4}+\frac{n}{4}}}{(2 \pi)^{\frac{1}{2}} K_{-\frac{n}{2}}\left(\frac{a}{\sqrt{\prod_{i=1}^{n-1}\left(1-v_{i}\right)}}\right)} \\
\times K_{-\frac{1}{2}-\frac{n}{2}}\left(\frac{a}{\sqrt{(1-x) \prod_{i=1}^{n-1}\left(1-v_{i}\right)}}\right), \\
n=2,3, \cdots
\end{array}\right.
$$

where $a>0$ and $K_{\mu}$ is the modified Bessel function of the third type (see e.g. Gradshteyn and Ryzhik (2014)). 
Similar to what we did for the Dirichlet process, we present the original definition of the normalized inverse Gaussian process as a proposition.

Proposition 7.5. A random probability measure $P$ is the normalized inverse Gaussian process with parameter $(a, H)$ if for any measurable partition $\left(A_{1}, \cdots, A_{n}\right)$ of $\mathbb{X}$, the random vector $\left(P\left(A_{1}\right), \cdots, P\left(A_{n}\right)\right)$ follows the normalized inverse Gaussian distribution with parameters $\left(a H\left(A_{1}\right), \cdots, a H\left(A_{n}\right)\right)$ given by the following form:

$$
\begin{aligned}
f\left(x_{1}, \cdots, x_{n}\right)= & \frac{e^{a} a^{n} \prod_{i=1}^{n} H\left(A_{i}\right)}{2^{\frac{n}{2}-1} \pi^{\frac{n}{2}}} \times K_{-\frac{n}{2}}\left(\sqrt{\sum_{i=1}^{n} \frac{\left(a H\left(A_{i}\right)\right)^{2}}{x_{i}}}\right) \\
& \times\left(\sum_{i=1}^{n} \frac{\left(a H\left(A_{i}\right)\right)^{2}}{x_{i}}\right)^{-\frac{n}{4}} \times \prod_{i=1}^{n} x_{i}^{-\frac{3}{2}} \times \mathbb{1}_{\mathbb{S}}\left(x_{1}, \cdots, x_{n}\right),
\end{aligned}
$$

where $\mathbb{S}$ is the simplex defined by (7.1).

Proof. We refer to Favaro et al. (2012) for the proof of the equivalence between Definition 7.4 and Proposition 7.5.

Definition 7.6 (Favaro et al. (2016)). $P$ is called the normalized generalized gamma process with parameters $\sigma \in(0,1), a>0$ and $H$, denoted by $P \sim \operatorname{NGG}(\sigma, a, H)$, if the finite dimensional joint distributions of the stick-breaking weights $\left\{v_{1}, v_{2}, \cdots\right\}$ are given by the following conditional distributions:

$$
\left\{\begin{array}{c}
f_{v_{1}}(x)=\frac{x^{-\sigma}(1-x)^{\sigma-1} e^{a}}{\Gamma(\sigma) \Gamma(1-\sigma)} \sum_{j=0}^{\infty} \frac{(1-\sigma)_{j}}{j !} \frac{a^{\frac{j}{\sigma}}}{(1-x)^{j}} \Gamma\left(1-\frac{j}{\sigma} ; \frac{a}{(1-x)^{\sigma}}\right), \\
f_{v_{n} \mid v_{1}, \cdots, v_{n-1}}(x)=\frac{\sigma \Gamma((n-1) \sigma) x^{-\sigma}(1-x)^{n \sigma-1}}{\Gamma(1-\sigma) \Gamma(n \sigma)} \\
\frac{\sum_{j=0}^{\infty} \frac{(1-n \sigma)_{j}}{j !} \frac{a^{\frac{j}{\sigma}}}{(1-x)^{j} \prod_{i=1}^{n-1}\left(1-v_{i}\right)^{j}} \Gamma\left(n-\frac{j}{\sigma} ; \frac{a}{(1-x)^{\sigma} \prod_{i=1}^{n-1}\left(1-v_{i}\right)^{\sigma}}\right)}{\sum_{j=0}^{\infty} \frac{(1-(n-1) \sigma)_{j}}{j !} \frac{a^{\frac{j}{\sigma}}}{\prod_{i=1}^{n-1}\left(1-v_{i}\right)^{j}} \Gamma\left(n-1-\frac{j}{\sigma} ; \frac{a}{\prod_{i=1}^{n-1}\left(1-v_{i}\right)^{\sigma}}\right)} \\
n=2,3, \cdots,
\end{array}\right.
$$

where $\Gamma(c, x)=\int_{x}^{\infty} u^{c-1} e^{-u} d u$ is the upper incomplete gamma function.

Definition 7.7. We call a random probability measure $P$ on $(\Omega, \mathcal{F})$ the generalized Dirichlet process with parameters $a>0, r \in \mathbb{N}^{+}$and $H$, denoted by $P \sim \operatorname{GDP}(a, r, H)$, if for any measurable partition $\left(A_{1}, \cdots, A_{n}\right)$ of $\mathbb{X}$, the joint density of $\left(P\left(A_{1}\right), \cdots, P\left(A_{n}\right)\right)$ is given by

$$
\begin{aligned}
f\left(x_{1}, \cdots, x_{n}\right)= & \frac{(r !)^{a}}{\prod_{i=1}^{n} \Gamma\left(r a_{i}\right)} \int_{0}^{\infty} t^{r a-1} e^{-r t}\left[\prod_{j=1}^{n} \Phi_{2}^{(r-1)}\left(a_{j} \mathbf{I}_{r-1} ; r a_{j} ; t x_{j} \mathbf{J}_{r-1}\right)\right] d t \\
& \times \prod_{i=1}^{n} x_{i}^{r a_{i}-1} \times \mathbb{1}_{\mathbb{S}}\left(x_{1}, \cdots, x_{n}\right)
\end{aligned}
$$


where $a_{i}=a H\left(A_{i}\right) ; \mathbf{I}_{r-1}=(1, \cdots, 1)^{T}, \mathbf{J}_{r-1}=(1, \cdots, r-1)$ are $r-1$ dimensional vectors and $\Phi_{2}^{N}(\mathbf{b} ; c ; \mathbf{x})$ is the confluent form of the fourth Lauricella hypergeometric function (see e.g. Exton (1976)), and $\mathbb{S}$ is the simplex defined by (7.1).

It is trivial to verify that the Dirichlet process is a special case of the generalized Dirichlet process with parameter $r=1$. Although the expression (7.7) looks very sophisticated, its mean, variance, and predictive distribution have been computed (see e.g. Lijoi et al. (2005b)). This process also admits a stick-breaking representation (e.g. Favaro et al. (2016)). However, the corresponding stick-breaking representation is more complicated to use for our study of the limiting theorems. So, we rather use this sophisticated finite dimensional distribution than the more sophisticated stick-breaking representation, which we omit.

For the Beta process, the stick-breaking representations are given in Paisley et al. (2010) and Teh et al. (2007). We use the former as our definition below.

Definition 7.8. A random measure $P$ is called the Beta process with parameters $a>0$, $\gamma>0, H$, denoted by $P \sim \operatorname{BP}(a, \gamma H)$, if it has the following representation:

$$
\left\{\begin{array}{l}
P=\sum_{i=1}^{\infty} \sum_{j=1}^{\kappa_{i}} w_{i, j} \delta_{\theta_{i, j}}, \quad \text { where } \\
w_{1, j}=v_{1, j}^{(1)}, \quad w_{i, j}=v_{i, j}^{(i)} \prod_{l=1}^{i-1}\left(1-v_{i, j}^{(l)}\right) \quad \text { for } \quad i=2,3, \cdots, \text { and } \quad j=1,2, \cdots,
\end{array}\right.
$$

where all variables are iid and $\kappa_{i} \stackrel{\text { iid }}{\sim} \operatorname{Poisson}(\gamma), v_{i, j}^{(l)} \stackrel{i i d}{\sim} \operatorname{Beta}(1, a), \theta_{i, j} \stackrel{\text { iid }}{\sim} H$ are mutually independent.

As we are presenting the functional central limit theorem of $P$, we need to recall the definition of the Brownian bridge process of parameter $H$ (see e.g. Kim and Bickel (2003) for more details).

Definition 7.9. Let $H$ be a measure on $(\mathbb{X}, \mathcal{X})$ and let $B_{H}^{o}=\left(B_{H}^{o}(\omega, A), \omega \in \Omega, A \in\right.$ $\mathcal{X})$ be a stochastic process (random measure) with parameter $A \in \mathcal{X}$. It is called the Brownian bridge with parameter $H$ if the following two conditions are satisfied.

(i) $B_{H}^{o}$ is Gaussian. Namely, for any elements $A_{1}, \cdots, A_{n} \in \mathcal{X}, B_{H}^{o}\left(A_{1}\right), \cdots, B_{H}^{o}\left(A_{n}\right)$ are jointly centered Gaussian random variables on the probability space $(\Omega, \mathcal{F}, \mathbb{P})$.

(ii) For any $A_{1}, A_{2} \in \mathcal{X}$, the covariance of $B_{H}^{o}\left(A_{1}\right)$ and $B_{H}^{o}\left(A_{2}\right)$ is given by

$$
\mathbb{E}\left[B_{H}^{o}\left(A_{1}\right) B_{H}^{o}\left(A_{2}\right)\right]=H\left(A_{1} \cap A_{2}\right)-H\left(A_{1}\right) H\left(A_{2}\right) .
$$

To state the functional central limit theorem we also need the space $D\left(\mathbb{R}^{d}\right)$ introduced in Section 3 of Bickel and Wichura (1971). The characteristics of the elements (functions) in $D\left(\mathbb{R}^{d}\right)$ are given by their continuity properties described as follows. For 
$1 \leq p \leq d$, let $R_{p}$ be one of the relations $<$ or $\geq$ and for $t=\left(t_{1}, \cdots, t_{d}\right) \in \mathbb{R}^{d}$ let $\mathcal{Q}_{R_{1}, \cdots, R_{d}}$ be the quadrant

$$
\mathcal{Q}_{R_{1}, \cdots, R_{d}}:=\left\{\left(s_{1}, \cdots, s_{d}\right) \in \mathbb{R}^{d}: s_{p} R_{p} t_{p}, 1 \leq p \leq d\right\} .
$$

Then, $x \in D\left(\mathbb{R}^{d}\right)$ if and only if (see e.g. Straf (1972)) for each $t \in \mathbb{R}^{d}$, the following two conditions hold: (i) $x_{\mathcal{Q}}=\lim _{s \rightarrow t, s \in \mathcal{Q}} x(s)$ exists for each of the $2^{d}$ quadrants $\mathcal{Q}=$ $\mathcal{Q}_{R_{1}, \cdots, R_{d}}(t)$ (namely, for all the combinations that $R_{1}=$ " $<$ ", or " $\geq$ ", $\cdots, R_{d}=$ " $<$ " or " $\geq "$ "), and (ii) $x(t)=x_{\mathcal{Q}>\ldots,>}$. In other words, $D\left(\mathbb{R}^{d}\right)$ is the space of functions that are "continuous from above with limits from below", which are similar to the space of the càdlàg (French word abbreviation for "right continuous with left limits") functions in one variable (i.e. $d=1$ ). The metric on $D\left(\mathbb{R}^{d}\right)$ is introduced as follows. Let $\Lambda=\left\{\lambda: \mathbb{R}^{d} \rightarrow \mathbb{R}^{d}: \lambda\left(t_{1}, \cdots, t_{d}\right)=\left(\lambda_{1}\left(t_{1}\right), \cdots, \lambda_{d}\left(t_{d}\right)\right)\right\}$, where each $\lambda_{p}: \mathbb{R} \rightarrow \mathbb{R}$ is continuous, strictly increasing and has limits at both infinities. Denote the Skorohod distance between $x, y \in D\left(\mathbb{R}^{d}\right)$ by

$$
d(x, y)=\inf \{\min (\|x-y \lambda\|,\|\lambda\|): \lambda \in \Lambda\},
$$

where $\|x-y \lambda\|=\sum_{n=1}^{\infty} \sup _{|t| \leq n}|x(t)-y(\lambda(t))|$ and $\|\lambda\|=\sum_{n=1}^{\infty} \sup _{|t| \leq n}|\lambda(t)-t|$.

Having introduced the metric space $D\left(\mathbb{R}^{d}\right)$ we can now explain the concept of weak convergence of a random measure on this space with respect to its Skorohod topology (the topology on $D\left(\mathbb{R}^{d}\right)$ induced by the Skorohod distance $d(x, y)$ ). Let $\mathbb{Q}_{a}: \Omega \times \mathcal{B}\left(\mathbb{R}^{d}\right) \rightarrow$ $[0,1]$ be a family of random probability measures depending on a parameter $a>0$ and let $\mathbb{B}: \Omega \times \mathcal{B}\left(\mathbb{R}^{d}\right) \rightarrow[0,1]$ be another random probability measure. Define

$$
\mathbb{Q}_{a}\left(t_{1}, \cdots, t_{d}\right)=\mathbb{Q}_{a}\left(\left(-\infty, t_{1}\right] \times \cdots \times\left(-\infty, t_{d}\right]\right), \quad\left(t_{1}, \cdots, t_{d}\right) \in \mathbb{R}^{d} .
$$

Definition 7.10. We say $\mathbb{Q}_{a}$ converges to $\mathbb{B}$ weakly on $D\left(\mathbb{R}^{d}\right)$ with respect to the Skorohod topology, denoted by $\mathbb{Q}_{a} \stackrel{\text { weakly }}{\rightarrow} \mathbb{B}$ in $D\left(\mathbb{R}^{d}\right)$, if for any bounded continuous (continuous with respect to Skorohod topology) functional $f: D\left(\mathbb{R}^{d}\right) \rightarrow \mathbb{R}$ we have

$$
\lim _{a \rightarrow \infty} \mathbb{E}\left[f\left(\mathbb{Q}_{a}(\cdot, \cdots, \cdot)\right)\right]=\mathbb{E}[f(\mathbb{B}(\cdot, \cdots, \cdot))] .
$$

Before the proofs of our results in the main body of the paper, we would like to give the mean and variance of the stick-breaking random measure $P$ as defined in (2.1)-(2.2). For any $A \in \mathcal{X}$,

$$
\begin{aligned}
\mathbb{E}(P(A)) & =\mathbb{E}\left[\sum_{i=1}^{\infty} w_{i} \delta_{\theta_{i}}(A)\right]=\sum_{i=1}^{\infty} \mathbb{E}\left(w_{i}\right) \mathbb{E}\left[\mathbb{1}_{A}\left(\theta_{i}\right)\right] \\
& =\sum_{i=1}^{\infty} \mathbb{E}\left(w_{i}\right) H(A)=\mathbb{E}\left[\sum_{i=1}^{\infty} w_{i}\right] H(A)=H(A),
\end{aligned}
$$

since $\sum_{i=1}^{\infty} w_{i}=1$ a.s. and

$$
\operatorname{Var}[P(A)]=\mathbb{E}\left[(P(A)-H(A))^{2}\right]=\mathbb{E}\left[\left(\sum_{i=1}^{\infty} w_{i}\left(\delta_{\theta_{i}}(A)-H(A)\right)\right)^{2}\right]
$$




$$
\begin{aligned}
= & \mathbb{E}\left[\sum_{i=1}^{\infty} w_{i}^{2}\left(\delta_{\theta_{i}}(A)-H(A)\right)^{2}\right] \\
& +2 \mathbb{E}\left[\sum_{1 \leq i<j<\infty} w_{i} w_{j}\left(\delta_{\theta_{i}}(A)-H(A)\right)\left(\delta_{\theta_{j}}(A)-H(A)\right)\right] \\
= & \mathbb{E}\left[\left(\delta_{\theta_{i}}(A)-H(A)\right)^{2}\right] \mathbb{E}\left[\sum_{i=1}^{\infty} w_{i}^{2}\right] \\
= & H(A)(1-H(A)) \mathbb{E}\left[\sum_{i=1}^{\infty} w_{i}^{2}\right] .
\end{aligned}
$$

\subsection{Proof of Proposition 3.1}

Proof of Proposition 3.1. Using the binomial expansion and using the fact that $v_{i} \in$ $[0,1]$ we have

$$
\begin{aligned}
\mathbb{E}\left[v_{i}^{n}\left(1-v_{i}\right)^{m}\right] & =\sum_{k=0}^{m}\left(\begin{array}{c}
m \\
k
\end{array}\right)(-1)^{k} \mathbb{E}\left[v_{i}^{n+k}\right] \\
& =\mathbb{E}\left[v_{i}^{n}\right]-m \mathbb{E}\left[v_{i}^{n+1}\right]+\cdots+(-1)^{m} \mathbb{E}\left[v_{i}^{m+n}\right] \\
& =\mathbb{E}\left[v_{1}^{n}\right]+O\left(\mathbb{E}\left[v_{1}^{n+1}\right]\right)=\mathbb{E}\left[v_{1}^{n}\right]+o\left(\mathbb{E}\left[v_{1}^{n}\right]\right),
\end{aligned}
$$

where the last equality follows from the assumption $\lim _{a \rightarrow \infty} \frac{\mathbb{E}\left[v_{1}^{n+1}\right]}{\mathbb{E}\left[v_{1}^{n}\right]}=0$ for all $n \in \mathbb{Z}_{+}$. Since $v_{i} \in[0,1]$ and since we assume that $v_{0}$ is not identically zero, we have $\mathbb{E}\left[\left(1-v_{i}\right)^{m}\right] \in$ $[0,1)$ and

$$
\begin{aligned}
\sum_{j=0}^{\infty}\left(\mathbb{E}\left[\left(1-v_{i}\right)^{m}\right]\right)^{j} & =\frac{1}{1-\mathbb{E}\left[\left(1-v_{i}\right)^{m}\right]} \\
& =\frac{1}{1-\sum_{j=0}^{m}\left(\begin{array}{c}
m \\
j
\end{array}\right)(-1)^{j} \mathbb{E}\left[v_{i}^{j}\right]} \\
& =\frac{1}{m \mathbb{E}\left[v_{1}\right]+\sum_{j=2}^{m}\left(\begin{array}{c}
m \\
j
\end{array}\right)(-1)^{j} \mathbb{E}\left[v_{i}^{j}\right]} \\
& =\frac{1}{m \mathbb{E}\left[v_{1}\right]}+o\left(\frac{1}{m \mathbb{E}\left[v_{1}\right]}\right),
\end{aligned}
$$

where the last equality also follows from the assumption $\lim _{a \rightarrow \infty} \frac{\mathbb{E}\left[v_{1}^{n+1}\right]}{\mathbb{E}\left[v_{1}^{n}\right]}=0$ for all $n \in \mathbb{Z}_{+}$. This proves (3.1)-(3.2).

Now we use (3.1)-(3.2) to show (3.3). Denote

$$
\mathcal{I}=\mathbb{E}\left[\sum_{1 \leq i_{1}<i_{2}<\cdots<i_{k}<\infty} w_{i_{1}}^{p_{1}} w_{i_{2}}^{p_{2}} \cdots w_{i_{k}}^{p_{k}}\right] .
$$


By the construction of the stick-breaking sequence $\left\{w_{i}\right\}_{i=1}^{\infty}$, we may rewrite $\mathcal{I}$ as

$$
\begin{aligned}
& \mathcal{I}= \\
& \sum_{1 \leq i_{1}<i_{2}<\cdots<i_{k}<\infty} \mathbb{E}\left[v_{i_{1}}^{p_{1}} \prod_{\ell_{1}=1}^{i_{1}-1}\left(1-v_{\ell_{1}}\right)^{p_{1}} \cdots v_{i_{m}}^{p_{m}} \prod_{\ell_{m}=1}^{i_{m}-1}\left(1-v_{\ell_{m}}\right)^{p_{m}} \cdots v_{i_{k}}^{p_{k}} \prod_{\ell_{k}=1}^{i_{k}-1}\left(1-v_{\ell_{k}}\right)^{p_{k}}\right] .
\end{aligned}
$$

Since $1 \leq i_{1}<i_{2}<\cdots<i_{k}<\infty$, we can rearrange $\mathcal{I}$ by putting $v$ 's with the same index together to obtain

$$
\begin{aligned}
& \mathcal{I}=\sum_{1 \leq i_{1}<i_{2}<\cdots<i_{k}<\infty} \mathbb{E}\left[v_{i_{1}}^{p_{1}}\left(1-v_{i_{1}}\right)^{p_{2: k}} \prod_{\ell_{1}=1}^{i_{1}-1}\left(1-v_{\ell_{1}}\right)^{p_{1: k}} v_{i_{2}}^{p_{2}}\left(1-v_{i_{2}}\right)^{p_{3: k}}\right. \\
& \prod_{\ell_{2}=i_{1}+1}^{i_{2}-1}\left(1-v_{\ell_{2}}\right)^{p_{2: k}} \cdots v_{i_{m}}^{p_{m}}\left(1-v_{i_{m}}\right)^{p_{m+1: k}} \prod_{\ell_{m}=i_{m-1}+1}^{i_{m}-1}\left(1-v_{\ell_{m}}\right)^{p_{m: k}} \\
& \left.\cdots v_{i_{k-1}}^{p_{k-1}}\left(1-v_{i_{k-1}}\right)^{p_{k}} \prod_{\ell_{k-1}=i_{k-2}+1}^{i_{k-1}-1}\left(1-v_{\ell_{k-1}}\right)^{p_{k}-1: k} v_{i_{k}}^{p_{k}} \prod_{\ell_{k}=i_{k-1}+1}^{i_{k}-1}\left(1-v_{\ell_{k}}\right)^{p_{k}}\right] .
\end{aligned}
$$

From the independence of $\left\{v_{1}, v_{2}, \cdots\right\}$ it follows

$$
\begin{aligned}
\mathcal{I}=\sum_{i_{1}=1}^{\infty} & \mathbb{E}\left[v_{i_{1}}^{p_{1}}\left(1-v_{i_{1}}\right)^{p_{2: k}}\right] \prod_{\ell_{1}=1}^{i_{1}-1} \mathbb{E}\left[\left(1-v_{\ell_{1}}\right)^{p_{1: k}}\right] \sum_{i_{2}=i_{1}+1}^{\infty} \mathbb{E}\left[v_{i_{2}}^{p_{2}}\left(1-v_{i_{2}}\right)^{p_{3: k}}\right] \\
& \prod_{\ell_{2}=i_{1}+1}^{i_{2}-1} \mathbb{E}\left[\left(1-v_{\ell_{2}}\right)^{p_{2: k}}\right] \ldots \sum_{i_{m}=i_{m-1}+1}^{\infty} \mathbb{E}\left[v_{i_{m}}^{p_{m}}\left(1-v_{i_{m}}\right)^{p_{m+1: k}}\right] \\
& \prod_{\ell_{m}=i_{m-1}+1}^{i_{m}-1} \mathbb{E}\left[\left(1-v_{\ell_{m}}\right)^{p_{m: k}}\right] \ldots \sum_{i_{k-1}=i_{k-2}+1}^{\infty} \mathbb{E}\left[v_{i_{k-1}}^{p_{k-1}}\left(1-v_{i_{k-1}}\right)^{p_{k}}\right] \\
& \prod_{\ell_{k-1}=i_{k-2}+1}^{i_{k-1}-1} \mathbb{E}\left[\left(1-v_{\ell_{k-1}}\right)^{p_{k-1: k}}\right] \sum_{i_{k}=i_{k-1}+1}^{\infty} \mathbb{E}\left[v_{i_{k}}^{p_{k}}\right] \prod_{\ell_{k}=i_{k-1}+1}^{i_{k}-1} \mathbb{E}\left[\left(1-v_{\ell_{k}}\right)^{p_{k}}\right] .
\end{aligned}
$$

Denoting the general factor in the above expression by

$$
\mathcal{S}_{m}=\sum_{i_{m}=i_{m-1}+1}^{\infty} \mathbb{E}\left[v_{i_{m}}^{p_{m}}\left(1-v_{i_{m}}\right)^{p_{m+1: k}}\right] \prod_{\ell_{m}=i_{m-1}+1}^{i_{m}-1} \mathbb{E}\left[\left(1-v_{\ell_{m}}\right)^{p_{m: k}}\right],
$$

for $m \in\{1,2, \cdots, k\}$, we can write

$$
\mathcal{I}=\mathcal{S}_{1} \mathcal{S}_{2} \cdots \mathcal{S}_{k}
$$

From the fact that $\left\{v_{1}, v_{2}, \cdots\right\}$ are identically distributed and by (3.1)-(3.2) we have 
for $m=1, \cdots, k$,

$$
\begin{aligned}
& \mathcal{S}_{m}=\mathbb{E}\left[v_{1}^{p_{m}}\left(1-v_{1}\right)^{p_{m+1: k}}\right] \sum_{i_{m}=i_{m-1}+1}^{\infty}\left(\mathbb{E}\left[\left(1-v_{1}\right)^{p_{m: k}}\right]\right)^{i_{m}-i_{m-1}-1} \\
& =\left(\mathbb{E}\left[v_{1}^{p_{m}}\right]+o\left(\mathbb{E}\left[v_{1}^{p_{m}}\right]\right)\right)\left(\frac{1}{p_{m: k} \mathbb{E}\left[v_{1}\right]}+o\left(\frac{1}{\mathbb{E}\left[v_{1}\right]}\right)\right) \\
& =\frac{\mathbb{E}\left[v_{1}^{p_{m}}\right]}{p_{m: k} \mathbb{E}\left[v_{1}\right]}+o\left(\frac{\mathbb{E}\left[v_{1}^{p_{m}}\right]}{p_{m: k} \mathbb{E}\left[v_{1}\right]}\right) .
\end{aligned}
$$

Substituting this estimate into (7.17), we see

$$
\begin{aligned}
\mathcal{I} & =\prod_{m=1}^{k}\left(\frac{\mathbb{E}\left[v_{1}^{p_{m}}\right]}{p_{m: k} \mathbb{E}\left[v_{1}\right]}+o\left(\frac{\mathbb{E}\left[v_{1}^{p_{m}}\right]}{p_{m: k} \mathbb{E}\left[v_{1}\right]}\right)\right) \\
& =\frac{\mathbb{E}\left[v_{1}^{p_{1}}\right] \cdots \mathbb{E}\left[v_{1}^{p_{m}}\right]}{p_{1: k} p_{2: k} \cdots p_{k: k}\left(\mathbb{E}\left[v_{1}\right]\right)^{k}}+o\left(\frac{\mathbb{E}\left[v_{1}^{p_{1}}\right] \cdots \mathbb{E}\left[v_{1}^{p_{m}}\right]}{\left(\mathbb{E}\left[v_{1}\right]\right)^{k}}\right) .
\end{aligned}
$$

This prove (3.3). If we take $p_{j}=2$ for all $j \in\{1, \cdots, k\}$, then $p_{1: k}=2 k$. The identity (3.4) is hence a straightforward consequence of (3.3).

\subsection{Proof of Proposition 3.2}

Since $v_{1}, v_{2}, \cdots$ are no longer identically distributed, the results established in the previous proof cannot be applied and we need some new computations. We shall still use the general method of moments. To this end, we need to recall some results about the hypergeometric functions and we refer to Aomoto et al. (2011) for further reading.

Definition 7.11. The hypergeometric function ${ }_{2} F_{1}(a, b, c ; x)$ (of parameters $a, b, c \in \mathbb{C}$, the complex plane) is defined by the series

$$
{ }_{2} F_{1}(a, b, c ; x)=\sum_{n=0}^{\infty} \frac{(a)_{n}(b)_{n}}{(c)_{n} n !} x^{n}
$$

for $|x|<1$, where $(q)_{n}$ is the Pochhammer symbol defined by

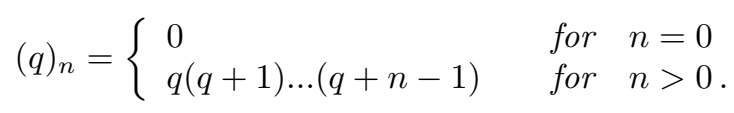

This function is defined for $|x|<1$ and may be extended to $x=1$ and/or $x=-1$ by continuation.

We need the following result obtained by Gauss: when $\operatorname{Re}(c-a-b)>0$ (real part of $c-a-b$ ), the hypergeometric function can be extended to $x=1$ and its value at this point is given by

$$
{ }_{2} F_{1}(a, b, c ; 1)=\frac{\Gamma(c) \Gamma(c-a-b)}{\Gamma(c-a) \Gamma(c-b)} .
$$


We introduce a variant of the hypergeometric function that will be needed in the following calculations.

Definition 7.12. For any $b<2, n \in \mathbb{N}^{+}, a>0, m>0, c>0$, define the increasing coefficient hypergeometric function ${ }_{2} Q_{1}((a, b), c, m, n ; x)$ by the series

$$
{ }_{2} Q_{1}((a, b), c, m, n ; x)=\sum_{k=0}^{\infty} \prod_{\ell=1}^{n-1}(a+b(k+\ell)) \frac{\left(\frac{a}{b}+1\right)_{k}(c)_{k}}{\left(\frac{a+m}{b}+1\right)_{k} k !} x^{k}
$$

for $|x|<1$ and we may extend the definition to $x=1$ and/or $x=-1$ by continuation. In the above product we use the convention that $\prod_{\ell=1}^{0} c_{\ell}=1$.

The next proposition describes a Gauss type result for the increasing coefficient hypergeometric function.

Proposition 7.13. Let $b<2, n \in \mathbb{N}^{+}, a>0, m>0, c>0$. Then, the increasing coefficient hypergeometric function can be extended to $x=1$ and its value at this point is given by

$$
{ }_{2} Q_{1}((a, b), c, m, n ; 1)=\prod_{\ell=1}^{n-1}(a+b \ell) \frac{a+m}{m-n b} .
$$

Proof. By (7.18) we have

$$
\begin{aligned}
& { }_{2} Q_{1}((a, b), c, m, n ; 1)=\prod_{\ell=1}^{n-1}(a+b \ell) \sum_{k=0}^{\infty} \prod_{i=1}^{k} \frac{a+(n+i-1) b}{a+i b+m} \\
& =\prod_{\ell=1}^{n-1}(a+b \ell){ }_{2} F_{1}\left(\frac{a}{b}+n, 1, \frac{a+m}{b}+1 ; 1\right)=\prod_{\ell=1}^{n-1}(a+b \ell) \frac{a+m}{m-n b},
\end{aligned}
$$

proving the proposition.

Now we are in the position of proving Proposition 3.2.

Proof of Proposition 3.2 Denote

$$
\mathcal{S}_{m}=\sum_{i_{m}=i_{m}+1}^{\infty} \mathbb{E}\left[v_{i_{m}}^{p_{m}}\left(1-v_{i_{m}}\right)^{p_{m+1: k}}\right] \prod_{\ell_{m}=i_{m-1}+1}^{i_{m}-1} \mathbb{E}\left[\left(1-v_{\ell_{m}}\right)^{p_{m: k}}\right],
$$

for $m \in\{1,2, \cdots, k\}$. Then we can write

$$
\mathcal{I}:=\mathbb{E}\left[\sum_{1 \leq i_{1}<i_{2}<\cdots<i_{k}<\infty} w_{i_{1}}^{p_{1}} w_{i_{2}}^{p_{2}} \cdots w_{i_{k}}^{p_{k}}\right]=\mathcal{S}_{1} \mathcal{S}_{2} \cdots \mathcal{S}_{k}
$$

We shall compute $\mathcal{I}$ by computing $\mathcal{S}_{m} \mathcal{S}_{m+1} \cdots \mathcal{S}_{k}$ recursively on $m=k, k-1, \cdots, 2,1$. First, by using (7.18) we have

$$
\mathcal{S}_{k}=\sum_{i_{k}=i_{k-1}+1}^{\infty} \frac{(1-b)_{p_{k}}}{\left(1+a+b\left(i_{k}-1\right)\right)_{p_{k}}} \prod_{\ell_{k}=i_{k-1}+1}^{i_{k}-1} \frac{\left(a+b \ell_{k}\right)_{p_{k}}}{\left(1+a+b\left(\ell_{k}-1\right)\right)_{p_{k}}}
$$




$$
\begin{aligned}
& =\frac{(1-b)_{p_{k}}}{\left(1+a+b i_{k-1}\right)_{p_{k}}}{ }_{2} F_{1}\left(\frac{a}{b}+i_{k-1}+1,1, \frac{a+p_{k}}{b}+i_{k-1}+1 ; 1\right) \\
& =\frac{(1-b)_{p_{k}}}{\left(p_{k}-b\right)\left(1+a+b i_{k-1}\right)_{p_{k}-1}} .
\end{aligned}
$$

Now we want to compute $\mathcal{S}_{m} \mathcal{S}_{m+1} \cdots \mathcal{S}_{k}$ assuming that we have already computed $\mathcal{S}_{m+1} \cdots \mathcal{S}_{k}$. To make thing clear we will explain how to compute $\mathcal{S}_{1} \cdots \mathcal{S}_{k}$ from the expression of $\mathcal{S}_{2} \cdots \mathcal{S}_{k}$. General case is similar. We assume

$$
\mathcal{S}_{2} \cdots \mathcal{S}_{k}=\frac{\left(a+b\left(i_{1}+1\right)\right) \cdots\left(a+b\left(i_{1}+k-2\right)\right)}{\left(1+a+b i_{1}\right)_{p_{2: k}-1}} \prod_{i=2}^{k} \frac{(1-b)_{p_{i}}}{p_{i: k}-(k-i+1) b} .
$$

Then, by Proposition 7.13

$$
\begin{aligned}
\mathcal{I} & =\mathcal{S}_{1} \mathcal{S}_{2} \cdots \mathcal{S}_{k}=(1-b)_{p_{1}} \prod_{i=2}^{k} \frac{(1-b)_{p_{i}}}{p_{i: k}-(k-i+1) b} \\
& \sum_{i=1}^{\infty} \frac{\left(a+b i_{1}\right) \cdots\left(a+b\left(i_{1}+k-2\right)\right)}{\left(1+a+b\left(i_{1}-1\right)\right)_{p_{1: k}}} \prod_{\ell_{1}=1}^{i_{1}-1} \frac{\left(a+b \ell_{1}\right)_{p_{1: k}}}{\left(1+a+b\left(\ell_{1}-1\right)\right)_{p_{1: k}}} \\
& =\frac{(1-b)_{p_{1}}}{(a+1)_{p_{1: k}}} \prod_{i=2}^{k} \frac{(1-b)_{p_{i}}}{p_{i: k}-(k-i+1) b}{ }_{2} Q_{1}\left((a, b), 1, p_{1: k}, k ; 1\right) \\
& =\frac{(1-b)_{p_{1}}}{(a+1)_{p_{1: k}}} \prod_{i=2}^{k} \frac{(1-b)_{p_{i}}}{p_{i: k}-(k-i+1) b} \prod_{\ell=1}^{k-1}(a+b \ell) \frac{a+p_{1: k}}{p_{1: k}-k b} \\
& =\frac{(a+b) \cdots(a+b(k-1))}{(1+a)_{p_{1: k}-1}} \prod_{i=1}^{k} \frac{(1-b)_{p_{i}}}{p_{i: k}-(k-i+1) b} \\
& =\frac{1}{(a+k b)(a+1)_{\left(p_{1: k}-1\right)}} \prod_{i=1}^{k} \frac{(1-b)_{p_{i}}(a+b i)}{p_{i: k}-(k-i+1) b} .
\end{aligned}
$$

This proves (3.5).

When $p_{j}=2$ for all $j \in\{1, \cdots, n\}$, we have easily

$$
\mathcal{I}=\mathbb{E}\left[\sum_{1 \leq i_{1}<i_{2}<\cdots<i_{n}<\infty} w_{i_{1}}^{2} w_{i_{2}}^{2} \cdots w_{i_{n}}^{2}\right]=\frac{(1-b)^{n}(a+b) \cdots(a+b(n-1))}{n !(a+1) \cdots(a+2 n-1)}
$$

proving (3.6).

\subsection{Proof of Proposition 3.3}

Proof of Proposition 3.3. By the stick-breaking representation of N-IG $(a, H)$ and the formula (3.471.9) in Gradshteyn and Ryzhik (2014), we find that the joint distribution 
of $\left\{v_{i}\right\}_{i=1}^{n}$ can be written as

$$
f\left(v_{1}, \cdots, v_{n}\right)=\frac{e^{a} a^{n+1}}{(2 \pi)^{\frac{n+1}{2}}} \prod_{i=1}^{n} v_{i}^{-\frac{1}{2}}\left(1-v_{i}\right)^{-\frac{n+3-i}{2}} \int_{0}^{\infty} t^{-\frac{n+3}{2}} e^{-\frac{t}{2}-\frac{a^{2}}{2 t \prod_{i=1}^{n}\left(1-v_{i}\right)}} d t .
$$

Thus, by using Fubini's theorem, we have

$$
\begin{aligned}
& \mathcal{I}=\mathbb{E}\left[\sum_{n=1}^{\infty} w_{n}^{p}\right]=\mathbb{E}\left[\sum_{n=1}^{\infty} v_{n}^{p} \prod_{i=1}^{n-1}\left(1-v_{i}\right)^{p}\right] \\
&= \sum_{n=1}^{\infty} \int_{0}^{\infty} \int_{0}^{1} \cdots \int_{0}^{1} \frac{e^{a} a^{n+1}}{(2 \pi)^{\frac{n+1}{2}}}\left(\prod_{i=1}^{n-1} v_{i}^{-\frac{1}{2}}\left(1-v_{i}\right)^{p-\frac{n+3-i}{2}}\right) v_{n}^{p-\frac{1}{2}}\left(1-v_{n}\right)^{-\frac{3}{2}} \\
& t^{-\frac{n+3}{2}} e^{-\frac{t}{2}-\frac{a^{2}}{2 t \prod_{i=1}^{n}\left(1-v_{i}\right)}} d v_{1} \cdots d v_{n} d t
\end{aligned}
$$

To evaluate the above multiple integral, we shall use the formula (3.471.2) in Gradshteyn and Ryzhik (2014):

$$
\int_{0}^{1}(1-v)^{\eta-1} v^{\mu-1} e^{-\frac{\beta}{1-v}} d v=\Gamma(\mu) \beta^{\frac{\eta-1}{2}} e^{-\frac{\beta}{2}} W_{\frac{1-2 \mu-\eta}{2}, \frac{\eta}{2}}(\beta),
$$

where $W$ is the Whittaker function. For large $\beta$, by the formula (9.227) in Gradshteyn and Ryzhik (2014), we have

$$
\int_{0}^{1}(1-v)^{\eta-1} v^{\mu-1} e^{-\frac{\beta}{1-v}} d v=\Gamma(\mu) \beta^{-\mu} e^{-\beta}\left(1+o\left(\frac{1}{\beta}\right)\right),
$$

as $\beta \rightarrow \infty$. In particular, when $\mu=\frac{1}{2}$, we have

$$
\int_{0}^{1}(1-v)^{\eta-1} v^{-\frac{1}{2}} e^{-\frac{\beta}{1-v}} d v=\Gamma\left(\frac{1}{2}\right) \beta^{-\frac{1}{2}} e^{-\beta}\left(1+o\left(\frac{1}{\beta}\right)\right) .
$$

Denote $\beta_{i}=\frac{a^{2}}{2 t\left(1-v_{n}\right) \prod_{\ell=1}^{i}\left(1-v_{\ell}\right)}$ for $i \in\{1, \cdots, n-1\}$. We rewrite $(7.22)$ as

$$
\begin{aligned}
\mathcal{I}= & \sum_{n=1}^{\infty} \int_{0}^{\infty} \int_{0}^{1} \cdots \int_{0}^{1} \frac{e^{a} a^{n+1}}{(2 \pi)^{\frac{n+1}{2}}} t^{-\frac{n+3}{2}} e^{-\frac{t}{2}}\left(\prod_{i=1}^{n-2} v_{i}^{-\frac{1}{2}}\left(1-v_{i}\right)^{p-\frac{n+3-i}{2}}\right) \\
& v_{n}^{p-\frac{1}{2}}\left(1-v_{n}\right)^{-\frac{3}{2}} \int_{0}^{1} v_{n-1}^{-\frac{1}{2}}\left(1-v_{n-1}\right)^{p-\frac{4}{2}} e^{-\frac{\beta_{n-2}}{\left(1-v_{n-1}\right)}} d v_{n-1} d v_{1} \cdots d v_{n-2} d v_{n} d t .
\end{aligned}
$$

Integrating with respect to $v_{n-1}$ by applying (7.25) yields

$$
\begin{aligned}
\mathcal{I}= & \sum_{n=1}^{\infty} \int_{0}^{\infty} \int_{0}^{1} \cdots \int_{0}^{1} \frac{e^{a} a^{n+1}}{(2 \pi)^{\frac{n+1}{2}}} \Gamma\left(\frac{1}{2}\right)\left(\frac{a^{2}}{2 t\left(1-v_{n}\right)}\right)^{-\frac{1}{2}} \\
& \left(1+o\left(\frac{1}{a}\right)\right) t^{-\frac{n+3}{2}} e^{-\frac{t}{2}}\left(1-v_{n}\right)^{-\frac{3}{2}} v_{n}^{p-\frac{1}{2}}\left(\prod_{i=1}^{n-2} v_{i}^{-\frac{1}{2}}\left(1-v_{i}\right)^{p-\frac{n+2-i}{2}}\right)
\end{aligned}
$$




$$
\int_{0}^{1} v_{n-2}^{-\frac{1}{2}}\left(1-v_{n-2}\right)^{p-\frac{4}{2}} e^{-\frac{\beta_{n-3}}{\left(1-v_{n-2}\right)}} d v_{1} \cdots d v_{n-2} d v_{n} d t
$$

We repeatedly apply the above procedure to integrate $v_{n-2}, v_{n-3}, \cdots, v_{1}$, each time using (7.25). After these computations we obtain

$$
\begin{aligned}
\mathcal{I}= & \sum_{n=1}^{\infty} \int_{0}^{\infty} \int_{0}^{1} \frac{e^{a} a^{n+1}}{(2 \pi)^{\frac{n+1}{2}}}\left(\Gamma\left(\frac{1}{2}\right)\left(\frac{a^{2}}{2 t\left(1-v_{n}\right)}\right)^{-\frac{1}{2}}\right)^{n-1}\left(1+o\left(\frac{1}{a}\right)\right) \\
& \times t^{-\frac{n+3}{2}} e^{-\frac{t}{2}} v_{n}^{p-\frac{1}{2}}\left(1-v_{n}\right)^{-\frac{3}{2}} d v_{n} d t \\
= & \sum_{n=1}^{\infty} \int_{0}^{\infty} \int_{0}^{1} \frac{e^{a} a^{2}}{2 \pi} v^{p-\frac{1}{2}}(1-v)^{\frac{n-4}{2}} e^{-\frac{a^{2}}{2 t(1-v)}} t^{-2} e^{-\frac{t}{2}}\left(1+o\left(\frac{1}{a}\right)\right) d v d t .
\end{aligned}
$$

Again by using Fubini's theorem and by the fact that $v \in(0,1)$, we take the sum to obtain

$$
\begin{aligned}
\mathcal{I} & =\int_{0}^{\infty} \int_{0}^{1} \frac{e^{a} a^{2}}{2 \pi} v^{p-\frac{1}{2}}\left(\sum_{n=1}^{\infty}(1-v)^{\frac{n-4}{2}}\right) e^{-\frac{a^{2}}{2 t(1-v)}} t^{-2} e^{-\frac{t}{2}}\left(1+o\left(\frac{1}{a}\right)\right) d v d t \\
& =\int_{0}^{\infty} \int_{0}^{1} \frac{e^{a} a^{2}}{2 \pi} v^{p-\frac{3}{2}}\left(1+(1-v)^{\frac{1}{2}}\right) e^{-\frac{a^{2}}{2 t(1-v)}} t^{-2} e^{-\frac{t}{2}}\left(1+o\left(\frac{1}{a}\right)\right) d v d t .
\end{aligned}
$$

Then, the result (3.7) is obtained by first applying (7.24) when we integrate the integral with respect to $v$, and then by applying the formula (3.471.9) in Gradshteyn and Ryzhik (2014) when we integrate $t$. Here, we also use the approximation that for fixed $\nu$ and for large $a, K_{\nu}(a)=\sqrt{\frac{\pi}{2}} a^{-1 / 2} e^{-a}\left(1+o\left(\frac{1}{a}\right)\right)$.

When $p=2$, we want to show that the leading coefficient in (3.7) is 1 . This needs some more delicate computations. First, we have

$$
\begin{aligned}
\mathcal{I}=\sum_{n=1}^{\infty} \int_{0}^{\infty} & \int_{0}^{1} \cdots \int_{0}^{1} \frac{e^{a} a^{n+1}}{(2 \pi)^{\frac{n+1}{2}}}\left(\prod_{i=1}^{n-1} v_{i}^{-\frac{1}{2}}\left(1-v_{i}\right)^{-\frac{n-1-i}{2}}\right) v_{n}^{\frac{3}{2}}\left(1-v_{n}\right)^{-\frac{3}{2}} \\
& \times t^{-\frac{n+3}{2}} e^{-\frac{t}{2}-\frac{a^{2}}{2 t \prod_{i=1}^{n}\left(1-v_{i}\right)}} d v_{1} \cdots d v_{n} d t .
\end{aligned}
$$

Notice the fact that the power of $v_{n-4}$ in the above integrand is $-\frac{3}{2}$ and to compute the integral with respect to $v_{n-4}$ we can use the following nice integral identity:

$$
\int_{0}^{1}(1-v)^{-\frac{3}{2}} v^{-\frac{1}{2}} e^{-\frac{\beta}{1-v}} d v=\Gamma\left(\frac{1}{2}\right) \beta^{-\frac{1}{2}} e^{-\beta} .
$$

After this integration with respect to $v_{n-4}$, we obtain an expression for $v_{n-5}$ which also has this form and we then integrate $v_{n-5}$ and so on. This procedure can continue until integrating $v_{1}$. Hence, we compute the integrals for $v_{n-4}$, and then for $v_{n-5}, \cdots$ and then for $v_{1}$ recursively to obtain

$$
\mathcal{I}=\sum_{n=1}^{\infty} \int_{0}^{\infty} \int_{0}^{1} \int_{0}^{1} \int_{0}^{1} \int_{0}^{1} \frac{e^{a} a^{5}}{(2 \pi)^{\frac{5}{2}}}\left(v_{n-3} v_{n-2} v_{n-1}\right)^{-\frac{1}{2}} v_{n}^{\frac{3}{2}}
$$




$$
\begin{aligned}
& \times\left(1-v_{n-3}\right)^{\frac{n-6}{2}}\left(1-v_{n-2}\right)^{\frac{n-5}{2}}\left(1-v_{n-1}\right)^{\frac{n-4}{2}}\left(1-v_{n}\right)^{\frac{n-7}{2}} \\
& \times e^{-\frac{a^{2}}{2 t\left(1-v_{n-3}\right)\left(1-v_{n-2}\right)\left(1-v_{n-1}\right)\left(1-v_{n}\right)}} t^{-\frac{7}{2}} e^{-\frac{t}{2}} d v_{n-3} d v_{n-2} d v_{n-1} d v_{n} d t .
\end{aligned}
$$

By Fubini's theorem and by the fact that $v_{i} \in(0,1)$, we can take the sum first [There is no need to sum up the index $n$ in $v_{n}, v_{n-1}, v_{n-2}, v_{n-3}$ since we can call them by other notations. But for consistency we still keep these notations.]

$$
\begin{aligned}
\mathcal{I}=\int_{0}^{\infty} & \int_{0}^{1} \int_{0}^{1} \int_{0}^{1} \int_{0}^{1} \frac{e^{a} a^{5}}{(2 \pi)^{\frac{5}{2}}}\left(v_{n-3} v_{n-2} v_{n-1}\right)^{-\frac{1}{2}} v_{n}^{\frac{3}{2}}\left(1-v_{n-3}\right)^{-\frac{5}{2}}\left(1-v_{n-2}\right)^{-\frac{4}{2}} \\
& \times\left(1-v_{n-1}\right)^{-\frac{3}{2}}\left(1-v_{n}\right)^{-\frac{6}{2}} \frac{1+\sqrt{\left(1-v_{n-3}\right)\left(1-v_{n-2}\right)\left(1-v_{n-1}\right)\left(1-v_{n}\right)}}{1-\left(1-v_{n-3}\right)\left(1-v_{n-2}\right)\left(1-v_{n-1}\right)\left(1-v_{n}\right)} \\
& \times e^{-\frac{a^{2}}{2 t\left(1-v_{n-3}\right)\left(1-v_{n-2}\right)\left(1-v_{n-1}\right)\left(1-v_{n}\right)}} t^{-\frac{7}{2}} e^{-\frac{t}{2}} d v_{n-3} d v_{n-2} d v_{n-1} d v_{n} d t .
\end{aligned}
$$

Now we integrate $t$ by using the formula (3.471.9) in Gradshteyn and Ryzhik (2014) to obtain

$$
\begin{aligned}
\mathcal{I}=\int_{0}^{1} & \int_{0}^{1} \int_{0}^{1} \int_{0}^{1} \frac{2 e^{a} a^{\frac{5}{2}}}{(2 \pi)^{\frac{5}{2}}}\left(v_{n-3} v_{n-2} v_{n-1}\right)^{-\frac{1}{2}} v_{n}^{\frac{3}{2}}\left(1-v_{n-3}\right)^{-\frac{5}{4}}\left(1-v_{n-2}\right)^{-\frac{3}{4}} \\
& \left(1-v_{n-1}\right)^{-\frac{1}{4}}\left(1-v_{n}\right)^{-\frac{7}{4}} \frac{1+\sqrt{\left(1-v_{n-3}\right)\left(1-v_{n-2}\right)\left(1-v_{n-1}\right)\left(1-v_{n}\right)}}{1-\left(1-v_{n-3}\right)\left(1-v_{n-2}\right)\left(1-v_{n-1}\right)\left(1-v_{n}\right)} \\
& K_{-\frac{5}{2}}\left(\frac{a}{\sqrt{\left(1-v_{n-3}\right)\left(1-v_{n-2}\right)\left(1-v_{n-1}\right)\left(1-v_{n}\right)}}\right) d v_{n-3} d v_{n-2} d v_{n-1} d v_{n} .
\end{aligned}
$$

Approximating the above modified Bessel function $K_{-\frac{5}{2}}$ of the third type by the formula (8.451.6) in Gradshteyn and Ryzhik (2014) we have

$$
\begin{aligned}
\mathcal{I}=\int_{0}^{1} & \int_{0}^{1} \int_{0}^{1} \int_{0}^{1} \frac{e^{a} a^{2}}{(2 \pi)^{2}}\left(v_{n-3} v_{n-2} v_{n-1}\right)^{-\frac{1}{2}} v_{n}^{\frac{3}{2}}\left(1-v_{n-3}\right)^{-\frac{2}{2}}\left(1-v_{n-2}\right)^{-\frac{1}{2}} \\
& \times\left(1-v_{n-1}\right)^{-\frac{0}{2}}\left(1-v_{n}\right)^{-\frac{3}{2}} \frac{1+\sqrt{\left(1-v_{n-3}\right)\left(1-v_{n-2}\right)\left(1-v_{n-1}\right)\left(1-v_{n}\right)}}{1-\left(1-v_{n-3}\right)\left(1-v_{n-2}\right)\left(1-v_{n-1}\right)\left(1-v_{n}\right)} \\
& \times e^{-\frac{a^{2}}{\sqrt{\left(1-v_{n-3}\right)\left(1-v_{n-2}\right)\left(1-v_{n-1}\right)\left(1-v_{n}\right)}}}\left(1+o\left(\frac{1}{a}\right)\right) d v_{n-3} d v_{n-2} d v_{n-1} d v_{n} .
\end{aligned}
$$

To evaluate the above integral we make the following variable substitutions.

$$
\left\{\begin{array}{l}
v_{n}=1-\frac{a^{2}}{\left(y_{0}+a\right)^{2}} ; \\
v_{n-1}=1-\frac{\left(y_{0}+a\right)^{2}}{\left(y_{0}+y_{1}+a\right)^{2}} ; \\
v_{n-2}=1-\frac{\left(y_{0}+y_{1}+a\right)^{2}}{\left(y_{0}+y_{1}+y_{2}+a\right)^{2}} ; \\
v_{n-3}=1-\frac{\left(y_{0}+y_{1}+y_{2}+a\right)^{2}}{\left(y_{0}+y_{1}+y_{2}+y_{3}+a\right)^{2}} .
\end{array}\right.
$$


The integral $\mathcal{I}$ can then be written as

$$
\begin{aligned}
\mathcal{I}=\int_{0}^{1} & \int_{0}^{1} \int_{0}^{1} \int_{0}^{1} \frac{e^{a} a^{2}}{(2 \pi)^{2}}\left(1-\frac{a^{2}}{\left(y_{0}+a\right)^{2}}\right)^{\frac{3}{2}}\left(1-\frac{\left(y_{0}+a\right)^{2}}{\left(y_{0}+y_{1}+a\right)^{2}}\right)^{-\frac{1}{2}} \\
& \times\left(1-\frac{\left(y_{0}+y_{1}+a\right)^{2}}{\left(y_{0}+y_{1}+y_{2}+a\right)^{2}}\right)^{-\frac{1}{2}}\left(1-\frac{\left(y_{0}+y_{1}+y_{2}+a\right)^{2}}{\left(y_{0}+y_{1}+y_{2}+y_{3}+a\right)^{2}}\right)^{-\frac{1}{2}} \\
& \times\left(\frac{\left(y_{0}+y_{1}+y_{2}+a\right)^{2}}{\left(y_{0}+y_{1}+y_{2}+y_{3}+a\right)^{2}}\right)^{-\frac{2}{2}}\left(\frac{\left(y_{0}+y_{1}+a\right)^{2}}{\left(y_{0}+y_{1}+y_{2}+a\right)^{2}}\right)^{-\frac{1}{2}} \\
& \times\left(\frac{\left(y_{0}+a\right)^{2}}{\left(y_{0}+y_{1}+a\right)^{2}}\right)^{-\frac{0}{2}}\left(\frac{a^{2}}{\left(y_{0}+a\right)^{2}}\right)^{-\frac{3}{2}} \frac{1+\frac{a}{\left(y_{0}+y_{1}+y_{2}+y_{3}+a\right)}}{1-\frac{a^{2}}{\left(y_{0}+y_{1}+y_{2}+y_{3}+a\right)^{2}}} \\
& \times \frac{2^{4} a^{2}}{\left(y_{0}+a\right)\left(y_{0}+y_{1}+a\right)\left(y_{0}+y_{1}+y_{2}+a\right)\left(y_{0}+y_{1}+y_{2}+y_{3}+a\right)^{3}} \\
& e^{-y_{0}-y_{1}-y_{2}-y_{3}-a}\left(1+o\left(\frac{1}{a}\right)\right) d y_{0} d y_{1} d y_{2} d y_{3} .
\end{aligned}
$$

When $a$ is large, we have the following asymptotics:

$$
\begin{aligned}
& 1-\frac{a^{2}}{\left(y_{0}+a\right)^{2}}=\frac{2 a y_{0}+y_{0}^{2}}{a^{2}+2 a y_{0}+y_{0}^{2}}=\frac{2 y_{0}}{a}+o\left(\frac{1}{a}\right) ; \\
& 1-\frac{\left(y_{0}+a\right)^{2}}{\left(y_{0}+y_{1}+a\right)^{2}}=\frac{2 y_{1}}{a}+o\left(\frac{1}{a}\right) ; \\
& 1-\frac{\left(y_{0}+y_{1}+a\right)^{2}}{\left(y_{0}+y_{1}+y_{2}+a\right)^{2}}=\frac{2 y_{2}}{a}+o\left(\frac{1}{a}\right) ; \\
& 1-\frac{\left(y_{0}+y_{1}+y_{2}+a\right)^{2}}{\left(y_{0}+y_{1}+y_{2}+y_{3}+a\right)^{2}}=\frac{2 y_{3}}{a}+o\left(\frac{1}{a}\right) ; \\
& \frac{a^{2}}{\left(y_{0}+a\right)^{2}}=\frac{\left(y_{0}+a\right)^{2}}{\left(y_{0}+y_{1}+a\right)^{2}}=\frac{\left(y_{0}+y_{1}+a\right)^{2}}{\left(y_{0}+y_{1}+y_{2}+a\right)^{2}} ; \\
& \quad=\frac{\left(y_{0}+y_{1}+y_{2}+a\right)^{2}}{\left(y_{0}+y_{1}+y_{2}+y_{3}+a\right)^{2}}=1+o\left(\frac{1}{a}\right) ; \\
& 1+\frac{a}{\left(y_{0}+y_{1}+y_{2}+y_{3}+a\right)}=2+o\left(\frac{1}{a}\right) ; \\
& 1-\frac{a^{2}}{\left(y_{0}+y_{1}+y_{2}+y_{3}+a\right)^{2}}=\frac{2\left(y_{0}+y_{1}+y_{2}+y_{3}\right)}{a}+o\left(\frac{1}{a}\right) ; \\
& \frac{2^{4} a^{2}}{\left(y_{0}+a\right)\left(y_{0}+y_{1}+a\right)\left(y_{0}+y_{1}+y_{2}+a\right)\left(y_{0}+y_{1}+y_{2}+y_{3}+a\right)^{3}}=\frac{2^{4}}{a^{4}}+o\left(\frac{1}{a^{4}}\right) .
\end{aligned}
$$

Substituting the above asymptotics into (7.31), we see that when $a$ is large the integral $\mathcal{I}$ is

$$
\mathcal{I}=\int_{0}^{1} \int_{0}^{1} \int_{0}^{1} \int_{0}^{1} \frac{e^{a} a^{2}}{(2 \pi)^{2}}\left(\frac{2 y_{0}}{a}\right)^{\frac{3}{2}}\left(\frac{2 y_{1}}{a}\right)^{-\frac{1}{2}}\left(\frac{2 y_{2}}{a}\right)^{-\frac{1}{2}}\left(\frac{2 y_{3}}{a}\right)^{-\frac{1}{2}}
$$




$$
\begin{aligned}
& \times \frac{a}{\left(y_{0}+y_{1}+y_{2}+y_{3}\right)} \frac{2^{4}}{a^{4}} e^{-y_{0}-y_{1}-y_{2}-y_{3}-a}\left(1+o\left(\frac{1}{a}\right)\right) d y_{0} d y_{1} d y_{2} d y_{3} \\
&=\int_{0}^{1} \int_{0}^{1} \int_{0}^{1} \int_{0}^{1} \frac{4}{a \pi^{2}} \frac{y_{0}^{\frac{3}{2}} y_{1}^{-\frac{1}{2}} y_{2}^{-\frac{1}{2}} y_{3}^{-\frac{1}{2}}}{y_{0}+y_{1}+y_{2}+y_{3}} \\
& \times e^{-y_{0}-y_{1}-y_{2}-y_{3}}\left(1+o\left(\frac{1}{a}\right)\right) d y_{0} d y_{1} d y_{2} d y_{3} .
\end{aligned}
$$

The above integral can be further evaluated by making the following variable substitutions:

$$
\left\{\begin{array}{l}
y_{0}=t_{0} ; \\
y_{0}+y_{1}=t_{1} \\
y_{0}+y_{1}+y_{2}=t_{2} ; \\
y_{0}+y_{1}+y_{2}+y_{3}=t_{3} .
\end{array}\right.
$$

With these substitutions, (7.32) can be written as

$$
\begin{aligned}
\mathcal{I}=\int_{0}^{\infty} \int_{0}^{t_{3}} & \int_{0}^{t_{2}} \int_{0}^{t_{1}} \frac{4}{a \pi^{2}} \frac{t_{0}^{\frac{3}{2}}\left(t_{1}-t_{0}\right)^{-\frac{1}{2}}\left(t_{2}-t_{1}\right)^{-\frac{1}{2}}\left(t_{3}-t_{2}\right)^{-\frac{1}{2}}}{t_{3}} \\
& \times e^{-t_{3}}\left(1+o\left(\frac{1}{a}\right)\right) d t_{0} d t_{1} d t_{2} d t_{3} .
\end{aligned}
$$

Now we integrate $t_{0}$ by letting $t_{0}=u t_{1}$,

$$
\begin{aligned}
\mathcal{I}=\int_{0}^{\infty} \int_{0}^{t_{3}} \int_{0}^{t_{2}} \int_{0}^{1} \frac{4}{a \pi^{2}} \frac{t_{1}\left(u t_{1}\right)^{\frac{3}{2}}\left(t_{1}-u t_{1}\right)^{-\frac{1}{2}}\left(t_{2}-t_{1}\right)^{-\frac{1}{2}}\left(t_{3}-t_{2}\right)^{-\frac{1}{2}}}{t_{3}} \\
\quad \times e^{-t_{3}}\left(1+o\left(\frac{1}{a}\right)\right) d u d t_{1} d t_{2} d t_{3} \\
=\int_{0}^{\infty} \int_{0}^{t_{3}} \int_{0}^{t_{2}} \frac{4}{a \pi^{2}} \frac{\Gamma\left(\frac{5}{2}\right) \Gamma\left(\frac{1}{2}\right)}{\Gamma\left(\frac{6}{2}\right)} \frac{t_{1}^{2}\left(t_{2}-t_{1}\right)^{-\frac{1}{2}}\left(t_{3}-t_{2}\right)^{-\frac{1}{2}}}{t_{3}} \\
\quad \times e^{-t_{3}}\left(1+o\left(\frac{1}{a}\right)\right) d t_{1} d t_{2} d t_{3} .
\end{aligned}
$$

Similarly, we integrate $t_{1}, t_{2}, t_{3}$ one by one in this order to obtain

$$
\begin{aligned}
\mathcal{I} & =\frac{4}{a \pi^{2}} \frac{\Gamma\left(\frac{5}{2}\right) \Gamma\left(\frac{1}{2}\right)}{\Gamma\left(\frac{6}{2}\right)} \frac{\Gamma\left(\frac{6}{2}\right) \Gamma\left(\frac{1}{2}\right)}{\Gamma\left(\frac{7}{2}\right)} \frac{\Gamma\left(\frac{7}{2}\right) \Gamma\left(\frac{1}{2}\right)}{\Gamma\left(\frac{8}{2}\right)} \Gamma\left(\frac{6}{2}\right)\left(1+o\left(\frac{1}{a}\right)\right) \\
& =\frac{1}{a}+o\left(\frac{1}{a}\right)
\end{aligned}
$$

completing the proof of (3.9).

To prove the results (3.8) and (3.10), we denote

$$
\mathcal{L}=\mathbb{E}\left[\sum_{1 \leq i_{1}<i_{2}<\cdots<i_{k}<\infty} w_{i_{1}}^{p_{1}} w_{i_{2}}^{p_{2}} \cdots w_{i_{k}}^{p_{k}}\right] .
$$


We have

$$
\begin{aligned}
\mathcal{L}= & \sum_{1 \leq i_{1}<i_{2}<\cdots<i_{k}<\infty} \mathbb{E}\left[v_{i_{1}}^{p_{1}}\left(1-v_{i_{1}}\right)^{p_{2: k}} \prod_{\ell_{1}=1}^{i_{1}-1}\left(1-v_{\ell_{1}}\right)^{p_{1: k}} v_{i_{2}}^{p_{2}}\left(1-v_{i_{2}}\right)^{p_{3: k}}\right. \\
& \times \prod_{\ell_{2}=i_{1}+1}^{i_{2}-1}\left(1-v_{\ell_{2}}\right)^{p_{2: k}} \cdots v_{i_{m}}^{p_{m}}\left(1-v_{i_{m}}\right)^{p_{m+1: k}} \prod_{\ell_{m}=i_{m-1}+1}^{i_{m}-1}\left(1-v_{\ell_{m}}\right)^{p_{m: k}} \\
& \left.\ldots v_{i_{k-1}}^{p_{k-1}}\left(1-v_{i_{k-1}}\right)^{p_{k}} \prod_{\ell_{k-1}=i_{k-2}+1}^{i_{k-1}-1}\left(1-v_{\ell_{k-1}}\right)^{p_{k}-1: k} v_{i_{k}}^{p_{k}} \prod_{\ell_{k}=i_{k-1}+1}^{i_{k}-1}\left(1-v_{\ell_{k}}\right)^{p_{k}}\right]
\end{aligned}
$$

Using the explicit form of the joint density of $v_{1}, \cdots, v_{i_{k}}$, we have

$$
\begin{aligned}
\mathcal{L}= & \sum_{1 \leq i_{1}<i_{2}<\cdots<i_{k}<\infty} \int_{0}^{\infty} \int_{0}^{1} \cdots \int_{0}^{1} \frac{e^{a} a^{i_{k}+1}}{(2 \pi)^{\frac{i_{k}+1}{2}}} t^{-\frac{i_{k}+3}{2}} e^{-\frac{t}{2}} e^{-\frac{a^{2}}{2 t \prod_{j=1}^{i_{k}}\left(1-v_{j}\right)}} \\
& \times \prod_{\ell_{1}=1}^{i_{1}-1} v_{\ell_{1}}^{-\frac{1}{2}}\left(1-v_{\ell_{1}}\right)^{p_{1: k}-\frac{i_{k}+3-\ell_{1}}{2}} v_{i_{1}}^{p_{1}-\frac{1}{2}}\left(1-v_{i_{1}}\right)^{p_{2: k}-\frac{i_{k}+3-i_{1}}{2}} \\
& \quad \prod_{\ell_{m}=i_{m-1}+1}^{i_{m}-1} v_{\ell_{m}}^{-\frac{1}{2}}\left(1-v_{\ell_{m}}\right)^{p_{m: k}-\frac{i_{k}+3-\ell_{m}}{2}} v_{i_{m}}^{p_{m}-\frac{1}{2}}\left(1-v_{i_{m}}\right)^{p_{m+1: k}-\frac{i_{k}+3-i_{m}}{2}} \\
& \ldots \prod_{\ell_{k}=i_{k-1}+1}^{i_{k}-1} v_{\ell_{k}}^{-\frac{1}{2}}\left(1-v_{\ell_{k}}\right)^{p_{k}-\frac{i_{k}+3-\ell_{k}}{2}} v_{i_{k}}^{p_{k}-\frac{1}{2}}\left(1-v_{i_{k}}\right)^{-\frac{3}{2}} d v_{1} \cdots d v_{i_{k}} d t . \quad(7.35)
\end{aligned}
$$

Notice that the integrals of $v_{i_{k-1}+1}, v_{i_{k-1}+2}, \cdots v_{i_{k}}$ with the sum of $i_{k}$ from $i_{k-1}+1$ to $\infty$ is the same form as (7.22). Thus, by the computation (7.26), we have

$$
\begin{aligned}
\mathcal{L}= & \sum_{1 \leq i_{1}<i_{2}<\cdots<i_{k}<\infty} \int_{0}^{\infty} \int_{0}^{1} \cdots \int_{0}^{1} \frac{e^{a} a^{i_{k}+1}}{(2 \pi)^{\frac{i_{k}+1}{2}}} t^{-\frac{i_{k}+3}{2}} e^{-\frac{t}{2}} e^{-\frac{a^{2}}{2 t\left(1-v_{i_{k}}\right) \Pi_{j=1}^{i_{k-1}\left(1-v_{j}\right)}}} \\
& \prod_{\ell_{1}=1}^{i_{1}-1} v_{\ell_{1}}^{-\frac{1}{2}}\left(1-v_{\ell_{1}}\right)^{p_{1: k}-\frac{i_{k}+3-\ell_{1}}{2}} v_{i_{1}}^{p_{1}-\frac{1}{2}}\left(1-v_{i_{1}}\right)^{p_{2: k}-\frac{i_{k}+3-i_{1}}{2}} \ldots \\
& \left(\prod_{\ell_{k-1}=i_{k-2}+1}^{i_{k-1}-1} v_{\ell_{k-1}}^{-\frac{1}{2}}\left(1-v_{\ell_{k-1}}\right)^{p_{k}-\frac{i_{k}+3-\ell_{k-1}}{2}} v_{i_{k-1}}^{p_{k-1}-\frac{1}{2}}\left(1-v_{i_{k-1}}\right)^{-\frac{3}{2}}\right. \\
& \left.\quad\left(\frac{1}{2}\right)\left(\frac{a^{2}}{2 t\left(1-v_{i_{k}}\right) \prod_{j=1}^{i_{k-1}}\left(1-v_{j}\right)}\right)^{i_{k}-i_{k-1}-1}\right)^{\left(1+o\left(\frac{1}{a}\right)\right) d v_{1} \cdots d v_{i_{k-1}} d v_{v_{i_{k}}} d t} \\
& \sum_{1 \leq i_{1}<i_{2}<\cdots<i_{k-1}<\infty i_{k}=i_{k-1}+1}^{\infty} \int_{0}^{\infty} \int_{0}^{1} \ldots \int_{0}^{1} \frac{e^{a} a^{i_{k-1}+2}}{(2 \pi)^{\frac{i_{k-1}+2}{2}}} t^{-\frac{i_{k-1}+4}{2}} e^{-\frac{t}{2}}
\end{aligned}
$$




$$
\begin{aligned}
& \prod_{\ell_{1}=1}^{i_{1}-1} v_{\ell_{1}}^{-\frac{1}{2}}\left(1-v_{\ell_{1}}\right)^{p_{1: k}-\frac{i_{k-1}+4-\ell_{1}}{2}} v_{i_{1}}^{p_{1}-\frac{1}{2}}\left(1-v_{i_{1}}\right)^{p_{2: k}-\frac{i_{k-1}+4-i_{1}}{2}} \cdots \\
& \prod_{\ell_{k-1}=i_{k-2}+1}^{i_{k-1}-1} v_{\ell_{k-1}}^{-\frac{1}{2}}\left(1-v_{\ell_{k-1}}\right)^{p_{k}-\frac{i_{k-1}+4-\ell_{k-1}}{2}} v_{i_{k-1}}^{p_{k-1}-\frac{1}{2}}\left(1-v_{i_{k-1}}\right)^{-\frac{4}{2}} \\
& v_{i_{k}}^{p_{k}}\left(1-v_{i_{k}}\right)^{\frac{i_{k}-i_{k-1}-4}{2}} e^{-\frac{a^{2}}{2 t\left(1-v_{i_{k}}\right) \Pi_{j=1}^{i_{k-1}\left(1-v_{j}\right)}}}\left(1+o\left(\frac{1}{a}\right)\right) d v_{i_{k}} d v_{1} \cdots d v_{i_{k-1}} d t
\end{aligned}
$$

By a similar calculation to that of (7.27),

$$
\begin{aligned}
\mathcal{L}= & \sum_{1 \leq i_{1}<i_{2}<\cdots<i_{k-1}<\infty} \int_{0}^{\infty} \int_{0}^{1} \cdots \int_{0}^{1} O\left(\frac{1}{a}\right) \frac{e^{a} a^{i_{k-1}+1}}{(2 \pi)^{\frac{i_{k-1}+1}{2}}} t^{-\frac{i_{k-1}+3}{2}} e^{-\frac{t}{2}} \\
& \times \prod_{\ell_{1}=1}^{i_{1}-1} v_{\ell_{1}}^{-\frac{1}{2}}\left(1-v_{\ell_{1}}\right)^{p_{1: k}-\frac{i_{k-1}+3-\ell_{1}}{2}} v_{i_{1}}^{p_{1}-\frac{1}{2}}\left(1-v_{i_{1}}\right)^{p_{2: k}-\frac{i_{k-1}+3-i_{1}}{2}} \\
& \ldots \prod_{\ell_{k-1}=i_{k-2}+1}^{i_{k-1}-1} v_{\ell_{k-1}}^{-\frac{1}{2}}\left(1-v_{\ell_{k-1}}\right)^{p_{k}-\frac{i_{k-1}+3-\ell_{k-1}}{2}} v_{i_{k-1}}^{p_{k-1}-\frac{1}{2}}\left(1-v_{i_{k-1}}\right)^{-\frac{3}{2}} \\
& \times e^{-\frac{a^{2}}{2 t \prod_{j=1}^{i_{k-1}\left(1-v_{j}\right)}} d v_{v_{k}} d v_{1} \cdots d v_{i_{k-1}} d t .}
\end{aligned}
$$

We can perform the analogous computations for $i_{k-1}, i_{k-2}, \cdots, i_{1}$ in this order repeatedly to obtain (3.8).

When $p_{1}=\cdots=p_{k}=2$, similar computations to that in the proof of (3.9) can be carried out to obtain (3.10).

\subsection{Proof of Proposition 3.4}

Proof of Proposition 3.4. By the identities $\Gamma(c, x)=e^{-x} x^{c} \int_{0}^{\infty} e^{-x u}(1+u)^{c-1} d u$ and $\sum_{j=0}^{\infty} \frac{(n)_{j}}{j !} x^{j}=(1-x)^{n}$, we can rewrite the joint density of stick-breaking weights $v_{1}, \cdots, v_{n}$ as

$$
\begin{aligned}
f\left(v_{1}, \cdots, v_{n}\right)= & \frac{a^{n} \sigma^{n-1}}{[\Gamma(1-\sigma)]^{n} \Gamma(n \sigma)} \prod_{i=1}^{n} v_{i}^{-\sigma}\left(1-v_{i}\right)^{-(n-i) \sigma-1} e^{-\frac{a}{\prod_{i=1}^{n}\left(1-v_{i}\right)^{\sigma}}} \\
& \times \int_{0}^{\infty}\left(1-(1+t)^{-\frac{1}{\sigma}}\right)^{n \sigma-1}(1+t)^{n-1} e^{-\frac{a t}{\prod_{i=1}^{n}\left(1-v_{i}\right)^{\sigma}}} d t .
\end{aligned}
$$

We make the substitution $t=\frac{\prod_{i=1}^{n}\left(1-v_{i}\right)^{\sigma} s}{a}$ in the above integral. Then, when $a$ is large, namely, when $t$ is small, we have

$$
(1+t)^{-\frac{1}{\sigma}} \asymp 1-\frac{t}{\sigma}=1-\frac{\prod_{i=1}^{n}\left(1-v_{i}\right)^{\sigma}}{\sigma a} s,
$$


where and throughout this paper we use $\mu \asymp \nu$ to represent $\lim \frac{\mu}{\nu}=1$.

The integral in (7.39) is then approximated by

$$
\int_{0}^{\infty}\left(1-(1+t)^{-\frac{1}{\sigma}}\right)^{n \sigma-1}(1+t)^{n-1} e^{-\frac{a t}{\prod_{i=1}^{n}\left(1-v_{i}\right)^{\sigma}}} d t \asymp \frac{\prod_{i=1}^{n}\left(1-v_{i}\right)^{n \sigma^{2}} \Gamma(n \sigma)}{\sigma^{n \sigma-1} a^{n \sigma}} .
$$

Thus, for large $a$, the joint density of $v_{1}, \cdots, v_{n}$ has the following asymptotics:

$$
f\left(v_{1}, \cdots, v_{n}\right) \asymp \frac{(a \sigma)^{n-n \sigma}}{[\Gamma(1-\sigma)]^{n}} \prod_{i=1}^{n} v_{i}^{-\sigma}\left(1-v_{i}\right)^{n \sigma^{2}-(n-i) \sigma-1} e^{-\frac{a}{\prod_{i=1}^{n}\left(1-v_{i}\right)^{\sigma}}} .
$$

Now the identities (3.11) and (3.12) follows from the same arguments as that in the proof of Proposition 3.3 and from the use of the following identity, which holds true for any $q \in \mathbb{R}$ :

$$
\begin{aligned}
\int_{0}^{1} x^{-\sigma}(1-x)^{q} e^{-\frac{a}{(1-x)^{\sigma}}} d x \\
\quad=\frac{e^{-a}}{a \sigma} \int_{0}^{\infty}\left(1-\left(\frac{a}{a+s}\right)^{\frac{1}{\sigma}}\right)^{-\sigma}\left(\frac{a}{a+s}\right)^{\frac{q+1}{\sigma}+1} e^{-s} d s \\
=e^{-a}(a \sigma)^{\sigma-1}\left(1+o\left(\frac{1}{a}\right)\right) \int_{0}^{1} s^{-\sigma} e^{-s} d s \\
=e^{-a}(a \sigma)^{\sigma-1} \Gamma(1-\sigma)\left(1+o\left(\frac{1}{a}\right)\right),
\end{aligned}
$$

where the last equality follows from the substitution $s=\frac{a}{(1-x)^{-\sigma}}-a$ and the following asymptotics:

$$
\left(\frac{a}{a+s}\right)^{\frac{1}{\sigma}}=\left(1-\frac{s}{a+s}\right)^{\frac{1}{\sigma}}=1-\frac{s}{\sigma(a+s)}+o\left(\frac{1}{a}\right)
$$

To obtain the exact asymptotics in the case when $\sigma=\frac{1}{m}$ and $p=p_{1}=\cdots=p_{k}=2$, we first prove (3.13) using the same argument as that in the proof of (3.9). The only differences are as follows. First, we integrate $v_{n-m}, v_{n-m-1}, \cdots, v_{1}$ recursively in this order by using (7.42). After these integrations, it remains to integrate the variables $v_{n-m+1}, \cdots, v_{n}$. This multiple integral is now evaluated simultaneously by using the substitution

$$
v_{n-i}=1-\left(\frac{a+y_{0}+\cdots+y_{i-1}}{a+y_{0}+\cdots+y_{i}}\right)^{\frac{1}{\sigma}}, \quad i=0, \cdots, m-1 .
$$

The identity (3.14) follows from (3.13) by the same argument as that in the proof of (3.10). 


\subsection{Proof of Proposition 3.5}

Proof of Proposition 3.5. When $P \sim \operatorname{GDP}(a, r, H)$, we will prove the weak convergence of $D_{a}$ in the part (i) of Theorem 4.15. Combining with the fact that $\operatorname{GDP}(a, r, H)$ also admits the general stick-breaking representation as in (2.1) and (2.2), we can obtain the desired results by using the same argument as that in the proof of (4.5) in Theorem 4.4 .

\subsection{Proof of Theorem 4.2}

Proof.

$$
\begin{aligned}
& \mathbb{P}(|P(A)-H(A)|>\epsilon) \leq \frac{\mathbb{E}\left[|P(A)-H(A)|^{m}\right]}{\epsilon^{m}} \frac{\mathbb{E}\left[\left|\sum_{i=1}^{\infty} w_{i}\left(\delta_{\theta_{i}}(A)-H(A)\right)\right|^{m}\right]}{\epsilon^{m}} \\
&= \sum \frac{c\left(p_{1}, \cdots, p_{k}\right)}{\epsilon^{m}} \prod_{i=1}^{k} \mathbb{E}\left[\left|\delta_{\theta_{i}}(A)-H(A)\right|^{p_{i}}\right] \\
& \times \mathbb{E}\left[\sum_{1 \leq i_{1}<i_{2}<\cdots<i_{k}<\infty} w_{i_{1}}^{p_{1}} w_{i_{2}}^{p_{2}} \cdots w_{i_{k}}^{p_{k}}\right]
\end{aligned}
$$

where the first sum is taken over all combinations of nonnegative integers $\left\{p_{1}, \cdots, p_{k}\right\}$ such that $k \in\{1, \cdots, m\}$ and $\sum_{i=1}^{k} p_{i}=m$ and where $c\left(p_{1}, \cdots, p_{k}\right)=\left(\begin{array}{c}m \\ p_{1}, \cdots, p_{k}\end{array}\right)=$ $\frac{m !}{p_{1} ! \cdots p_{k} !}$ are the corresponding combinatorial coefficients. For any combination of $\left\{p_{1}, \cdots, p_{k}\right\}$, if there exists $i \in\{1, \cdots, k\}$ such that $p_{i}=1$, then the product in (7.43) will be 0 due to the fact that

$$
\mathbb{E}\left[\delta_{\theta_{i}}\left(A_{i}\right)-H\left(A_{i}\right)\right]=\mathbb{E}\left[\mathbb{1}_{A_{i}}\left(\theta_{i}\right)\right]-H\left(A_{i}\right)=\int_{A_{i}} d H-H\left(A_{i}\right)=0 .
$$

That is to say, $p_{i} \geq 2$ for all $i$ and thus $k \leq \frac{m}{2}$.

First, assume $P$ is one of $\operatorname{DP}(a, H), \operatorname{PDP}(a, b, H), \mathrm{N}-\mathrm{IG}(a, H), \operatorname{NGG}(\sigma, a, H), \operatorname{GDP}(a, r, H)$. We choose $m=\left\lfloor\frac{4}{\tau}\right\rfloor$, where $\lfloor x\rfloor$ is the smallest integer that is greater than or equal to $x$. Then, from Propositions 3.2-3.5, we have

$$
\sum \mathbb{E}\left[\sum_{1 \leq i_{1}<i_{2}<\cdots<i_{k}<\infty} w_{i_{1}}^{p_{1}} w_{i_{2}}^{p_{2}} \cdots w_{i_{k}}^{p_{k}}\right] \asymp \frac{1}{a^{m-k}} \leq \frac{1}{a^{\frac{m}{2}}}=\frac{1}{N^{\frac{m \tau}{2}}} \leq \frac{1}{N^{2}}
$$

If $p \sim \operatorname{SPG}\left(\mu_{a}, H\right)$, then we can choose $m$ such that for all $1 \leq k \leq m / 2$,

$$
\sum_{1 \leq i \leq k, p_{1}+\cdots+p_{k}=m} q_{p_{i}}-k q_{1} \geq \frac{m\left(q_{2}-q_{1}\right)}{2} \geq \frac{2}{\tau} .
$$


Then, from Proposition 3.1,

$$
\sum \mathbb{E}\left[\sum_{1 \leq i_{1}<i_{2}<\cdots<i_{k}<\infty} w_{i_{1}}^{p_{1}} w_{i_{2}}^{p_{2}} \cdots w_{i_{k}}^{p_{k}}\right] \asymp \frac{1}{a^{\sum_{i=1}^{k} q_{p_{i}}-k q_{1}}} \leq \frac{1}{N^{2}} .
$$

Since the series $\sum_{N=1}^{\infty} \frac{1}{N^{2}}$ converges, it follows

$$
\sum_{N=1}^{\infty} \mathbb{P}(|P(A)-H(A)|>\epsilon)<\infty
$$

for any of the processes presented in the theorem. This implies (4.2) by the Borel-Cantelli lemma.

\subsection{Proof of Theorem 4.4}

Before we proceed to the proof of Theorem 4.4, we need a preparatory result about the joint moments of multivariate normal distribution. To state this result, we introduce the following notations. Let $n$ be a positive integer and let $\vec{p}=\left(p_{i j}, 1 \leq i<j \leq n\right)$ be a multi-index. Denote

$$
|\vec{p}|=\sum_{1 \leq i<j \leq n} p_{i j}
$$

and denote

$$
|\vec{p}|_{m}= \begin{cases}\sum_{j>1} p_{1 j} & \text { when } m=1, \\ \sum_{j>m} p_{m j}+\sum_{i<m} p_{i m} & \text { when } m=2, \cdots, n-1, \\ \sum_{i<n} p_{i n} & \text { when } m=n .\end{cases}
$$

The following proposition is about the joint moments of Gaussian random variables. Similar or more general results may be found in literature under the terminology of "Feynman diagram" (e.g. Hu (2017, Theorem 5.7) and references therein). But we could not find the exact result we need. So, we give the following proposition.

Proposition 7.14. Let the random vector $\left(X_{1}, X_{2}, \cdots, X_{n}\right)$ follow a multivariate normal distribution $N_{n}(0, \Sigma)$, where $\Sigma=\left(\sigma_{i j}=\mathbb{E}\left(X_{i} X_{j}\right)\right)_{1 \leq i, j \leq n}$ and $\sigma_{i i}=\sigma_{i}^{2}$. For any nonnegative integers $r_{1}, \cdots, r_{n}$, the joint $\left(r_{1}, \cdots, r_{n}\right)$ moments of $\left(X_{1}, X_{2}, \cdots, X_{n}\right)$ is given by the following formulas.

(i) When $\sum_{\ell=1}^{n} r_{\ell}$ is an odd integer, we have

$$
\mathbb{E}\left[X_{1}^{r_{1}} \cdots X_{n}^{r_{n}}\right]=0 .
$$

(ii) When $\sum_{\ell=1}^{n} r_{\ell}$ is an even integer, we have (we use the convention that $0^{0}=1$ )

$$
\mathbb{E}\left[X_{1}^{r_{1}} \cdots X_{n}^{r_{n}}\right]
$$




$$
=\sum_{\vec{p} \in \mathcal{C}} \frac{r_{1} ! \cdots r_{n} ! \prod_{m=1}^{n} \sigma_{m}^{r_{m}-|\vec{p}|_{m}} \prod_{1 \leq i<j \leq n} \sigma_{i j}^{p_{i j}}}{\prod^{\frac{|r|}{2}-|\vec{p}|} \prod_{m=1}^{n}\left(\left(r_{m}-|\vec{p}|_{m}\right) / 2\right) ! \prod_{1 \leq i<j \leq n} p_{i j} !},
$$

where $|r|=r_{1}+\cdots+r_{n}$ and

$$
\begin{array}{r}
\mathcal{C}=\left\{\vec{p}=\left\{p_{i j}, 1 \leq i<j \leq n\right\} ; \quad 0 \leq p_{i j} \leq r_{i} \wedge r_{j},\right. \\
\left.r_{m}-|\vec{p}|_{m} \quad m=1, \cdots, n, \text { are all even }\right\} .
\end{array}
$$

Proof. We shall use the moment generating function to prove it. On one hand, we see

$$
\mathbb{E}\left[e^{\sum_{\ell=1}^{n} t_{\ell} X_{\ell}}\right]=\sum_{r_{1}, \cdots, r_{n}=0}^{\infty} \frac{t_{1}^{r_{1}} \cdots t_{n}^{r_{n}}}{r_{1} ! \cdots r_{n} !} \mathbb{E}\left[X_{1}^{r_{1}} \cdots X_{n}^{r_{n}}\right] .
$$

On the other hand, by the moment generating function formula for multivariate normal variables, we have

$$
\begin{aligned}
\mathbb{E}\left[e^{\sum_{\ell=1}^{n} t_{\ell} X_{\ell}}\right]=e^{\frac{1}{2} \mathbb{E}\left[\left(\sum_{\ell=1}^{n} t_{\ell} X_{\ell}\right)^{2}\right]} \\
=\exp \left\{\frac{1}{2} \sum_{\ell=1}^{n} \mathbb{E}\left[\left(t_{\ell} X_{\ell}\right)^{2}\right]+\sum_{1 \leq i<j \leq n} \mathbb{E}\left[t_{i} t_{j} X_{i} X_{j}\right]\right\} \\
=\prod_{\ell=1}^{n} \exp \left\{\frac{t_{\ell}^{2} \sigma_{\ell}^{2}}{2}\right\} \prod_{1 \leq i<j \leq n} \exp \left\{t_{i} t_{j} \sigma_{i j}\right\} \\
=\sum_{\substack{0 \leq p_{l}, p_{i j}<\infty \\
1 \leq \ell \leq n \\
1 \leq i<j \leq n}} \prod_{\ell=1}^{n} \frac{\left(t_{\ell} \sigma_{\ell}\right)^{2 p_{\ell}}}{2^{p_{\ell}} p_{\ell} !} \prod_{1 \leq i<j \leq n} \frac{\left(t_{i} t_{j} \sigma_{i j}\right)^{p_{i j}}}{p_{i j} !} \\
=\sum_{\substack{0 \leq p_{l}, p_{i j}<\infty \\
1 \leq \ell \leq n \\
1 \leq i<j \leq n}} \frac{t_{1}^{2 p_{1}+|\vec{p}|_{1}} \ldots t_{n}^{2 p_{n}+|\vec{p}|_{m}}}{2^{p_{1}+\cdots+p_{n}}} \prod_{\ell=1}^{n} \frac{\sigma_{\ell}^{2 p_{\ell}}}{p_{\ell} !} \prod_{1 \leq i<j \leq n} \frac{\sigma_{i j}^{p_{i j}}}{p_{i j} !} .
\end{aligned}
$$

Comparing the coefficients of $t_{1}^{r_{1}} \cdots t_{n}^{r_{n}}$ of the two representations (7.50) and (7.52) we obtain

$$
\mathbb{E}\left[X_{1}^{r_{1}} \cdots X_{n}^{r_{n}}\right]=\frac{r_{1} ! \cdots r_{n} !}{2^{p_{1}+\cdots+p_{n}}} \prod_{\ell=1}^{n} \frac{\sigma_{\ell}^{2 p_{\ell}}}{p_{\ell} !} \prod_{1 \leq i<j \leq n} \frac{\sigma_{i j}^{p_{i j}}}{p_{i j} !},
$$

where $p_{\ell}, p_{i j}, 1 \leq \ell \leq n, 1 \leq i<j \leq n$ satisfies the relation $r_{\ell}=2 p_{\ell}+|\vec{p}|_{\ell}$ for $\ell=1, \cdots, n$, which implies that $\sum_{\ell=1}^{n} r_{\ell}=2\left(\sum_{\ell=1}^{n} p_{\ell}+\sum_{1 \leq i, j \leq n} p_{i j}\right)$ is an even number. This also proves that $\mathbb{E}\left[X_{1}^{r_{1}} \cdots X_{n}^{r_{n}}\right]=0$ when $\sum_{\ell=1}^{n} r_{\ell}$ is an odd integer. This proves part (i) of the proposition. When $\sum_{\ell=1}^{n} r_{\ell}$ is even, by the fact that $\left\{p_{1}, \cdots, p_{n}\right\}$ are nonnegative integers and by the relationship $p_{\ell}=\frac{r_{\ell}-|\vec{p}| \ell}{2}$ for $\ell=1, \cdots, n$, one see the summation in (7.53) is over the set $\mathcal{C}$ defined by $(7.49)$. 
Now, we are in the position to prove Theorem 4.4.

Proof of Theorem 4.4. We first prove the part (i) of this theorem.

For any $A \in \mathcal{X}$, the variance of $P(A)$ is given by (7.13). Using Proposition 3.1, we have

$$
\operatorname{Var}(P(A))=H(A)(1-H(A))\left(\frac{\mathbb{E}\left[v_{1}^{2}\right]}{2 \mathbb{E}\left[v_{1}\right]}+o\left(\frac{\mathbb{E}\left[v_{1}^{2}\right]}{\mathbb{E}\left[v_{1}\right]}\right)\right)
$$

From the definition of $D_{a}(A)$ we have

$$
D_{a}(A)=\left[H(A)(1-H(A))\left(\frac{\mathbb{E}\left[v_{1}^{2}\right]}{2 \mathbb{E}\left[v_{1}\right]}+o\left(\frac{\mathbb{E}\left[v_{1}^{2}\right]}{\mathbb{E}\left[v_{1}\right]}\right)\right)\right]^{-\frac{1}{2}}(P(A)-H(A)) .
$$

By the Cramér-Wold theorem (e.g. Billingsley (1995, Theorem 29.4)), to show (4.5) it is sufficient to show that for any $\left(t_{1}, \cdots, t_{n}\right) \in \mathbb{R}^{n}$

$$
\sum_{i=1}^{n} t_{i} D_{a}\left(A_{i}\right) \stackrel{d}{\rightarrow} \sum_{i=1}^{n} t_{i} X_{i}
$$

where and throughout the remaining part of the paper $\left(X_{1}, \cdots, X_{n}\right)$ are jointly Gaussian with mean zero and covariance given by (4.6). For any positive integer $n$ and a nonnegative integer sequence $\left\{r_{i}\right\}_{i=1}^{n}$, consider the joint moments of $D_{a}\left(A_{1}\right), D_{a}\left(A_{2}\right), \cdots, D_{a}\left(A_{n}\right)$ :

$$
\begin{aligned}
& \mathbb{E}\left[\prod_{i=1}^{n} D_{a}^{r_{i}}\left(A_{i}\right)\right] \\
& =\prod_{i=1}^{n}\left[H\left(A_{i}\right)\left(1-H\left(A_{i}\right)\right)\left(\frac{\mathbb{E}\left[v_{1}^{2}\right]}{2 \mathbb{E}\left[v_{1}\right]}+o\left(\frac{\mathbb{E}\left[v_{1}^{2}\right]}{\mathbb{E}\left[v_{1}\right]}\right)\right)\right]^{-\frac{r_{i}}{2}} \mathbb{E}\left[\prod_{i=1}^{n}\left(P\left(A_{i}\right)-H\left(A_{i}\right)\right)^{r_{i}}\right] \\
& =\prod_{i=1}^{n}\left[H\left(A_{i}\right)\left(1-H\left(A_{i}\right)\right)\left(\frac{\mathbb{E}\left[v_{1}^{2}\right]}{2 \mathbb{E}\left[v_{1}\right]}+o\left(\frac{\mathbb{E}\left[v_{1}^{2}\right]}{\mathbb{E}\left[v_{1}\right]}\right)\right)\right]^{-\frac{r_{i}}{2}} \times \\
& \mathbb{E}\left[\prod_{i=1}^{n}\left(\sum_{j_{i}=1}^{\infty} w_{j_{i}}\left(\delta_{\theta_{j_{i}}}\left(A_{i}\right)-H\left(A_{i}\right)\right)\right)^{r_{i}}\right]
\end{aligned}
$$

We expand the product of the infinite sums inside the above last expectation as

$$
\begin{aligned}
\mathbb{E}[ & \left.\prod_{i=1}^{n}\left(\sum_{j_{i}=1}^{\infty} w_{j_{i}}\left(\delta_{\theta_{j_{i}}}\left(A_{i}\right)-H\left(A_{i}\right)\right)\right)^{r_{i}}\right] \\
& =\sum C\left(q ; s_{1}, \cdots, s_{n}, s_{1,1}, \cdots, s_{q, n}\right) I\left(q ; s_{1}, \cdots, s_{n}, s_{1,1}, \cdots, s_{q, n}\right)
\end{aligned}
$$

where the sum is over all the nonnegative integers $\left\{q, s_{1}, \cdots, s_{n} ; s_{j, i}: j \in\{1, \cdots, q\} ; i \in\right.$ $\{1, \cdots, n\}\}$ satisfying 
(i) $s_{j}=\sum_{i=1}^{n} s_{j, i} \geq 1$ for all $j \in\{1, \cdots, q\}$

(ii) $\quad r_{i}=\sum_{j=1}^{q} s_{j, i} \geq 1$ for all $i \in\{1, \cdots, n\}$

(iii) $1 \leq q \leq \sum_{i=1}^{n} r_{i}$;

(iv) $\quad \sum_{j=1}^{q} s_{j}=\sum_{i=1}^{n} r_{i}$

(v) $C\left(q ; s_{1}, \cdots, s_{n}, s_{1,1}, \cdots, s_{q, n}\right)$ are some constants (that are found later on) depending on $q ; s_{1}, \cdots, s_{n}, s_{1,1}, \cdots, s_{q, n}$ and

$$
\begin{gathered}
I\left(q ; s_{1}, \cdots, s_{n}, s_{1,1}, \cdots, s_{q, n}\right) \\
:=\mathbb{E}\left[\sum_{1 \leq e_{1}<\cdots<e_{q}<\infty} \prod_{j=1}^{q} w_{e_{j}}^{s_{j}}\left(\delta_{\theta_{e_{j}}}\left(A_{1}\right)-H\left(A_{1}\right)\right)^{s_{j, 1}}\right. \\
\left.\cdots\left(\delta_{\theta_{e_{j}}}\left(A_{n}\right)-H\left(A_{n}\right)\right)^{s_{j, n}}\right] .
\end{gathered}
$$

With these notations, we can write (7.54) as

$$
\begin{gathered}
\mathbb{E}\left[\prod_{i=1}^{n} D_{a}^{r_{i}}\left(A_{i}\right)\right]=\sum \prod_{i=1}^{n}\left[H\left(A_{i}\right)\left(1-H\left(A_{i}\right)\right)\left(\frac{\mathbb{E}\left[v_{1}^{2}\right]}{2 \mathbb{E}\left[v_{1}\right]}+o\left(\frac{\mathbb{E}\left[v_{1}^{2}\right]}{\mathbb{E}\left[v_{1}\right]}\right)\right)\right]^{-\frac{r_{i}}{2}} \\
C\left(q ; s_{1}, \cdots, s_{n}, s_{1,1}, \cdots, s_{q, n}\right) I\left(q ; s_{1}, \cdots, s_{n}, s_{1,1}, \cdots, s_{q, n}\right) .
\end{gathered}
$$

We will divide the discussion of the limit as $a \rightarrow \infty$ of the terms in (7.56) into three cases according to the indice $\left\{q ; s_{1}, \cdots, s_{n} ; s_{j, i}: j \in\{1, \cdots, q\} ; i \in\{1, \cdots, n\}\right\}$ appeared in (7.56) satisfying (i)-(iv).

Case 1: There exists at least one $j \in\{1, \cdots, q\}$ such that $s_{j}=1$ or there exists at least one pair $(j, k)$ such that $s_{j, k}=1$.

From the fact that

$$
\mathbb{E}\left[\delta_{\theta_{i}}\left(A_{i}\right)-H\left(A_{i}\right)\right]=\mathbb{E}\left[\mathbb{1}_{A_{i}}\left(\theta_{i}\right)\right]-H\left(A_{i}\right)=\int_{A_{i}} d H-H\left(A_{i}\right)=0,
$$

we see that in this case the corresponding terms in the sum of $I\left(q ; s_{1}, \cdots, s_{n}, s_{1,1}, \cdots, s_{q, n}\right)$ are identically equal to 0 .

Case 2 All $s_{j} \geq 2, \sum_{i=1}^{n} r_{i}$ is odd or $\sum_{i=1}^{n} r_{i}$ is even but $\frac{\sum_{i=1}^{n} r_{i}}{2}>q$.

We substitute (7.55) into (7.56) and we consider the expectations of $v_{1}$ 's. By Proposition 3.1, when excluding the terms discussed in Case 1 the remaining terms corresponding to this case have the following asymptotics

$$
O\left(\left[\frac{\mathbb{E}\left[v_{1}^{2}\right]}{\mathbb{E}\left[v_{1}\right]}\right]^{-\frac{\sum_{i=1}^{n} r_{i}}{2}} \frac{\prod_{j=1}^{q} \mathbb{E}\left[v_{1}^{s_{j}}\right]}{\left(\mathbb{E}\left[v_{1}\right]\right)^{q}}\right)=O\left(\frac{\left(\mathbb{E}\left[v_{1}\right]\right)^{\frac{\sum_{j=1}^{q} s_{j}}{2}-q} \prod_{j=1}^{q} \mathbb{E}\left[v_{1}^{s_{j}}\right]}{\left(\mathbb{E}\left[v_{1}^{2}\right]\right)^{\frac{\sum_{j=1}^{q} s_{j}}{2}}}\right)
$$


From the assumption (4.4), it follows that when $\sum_{i=1}^{n} r_{i}$ is even and when $q<\frac{\sum_{i=1}^{n} r_{i}}{2}$, the expectation of the terms in the sum of $I\left(q ; s_{1}, \cdots, s_{n}, s_{1,1}, \cdots, s_{q, n}\right)$ will converge to 0 as $a \rightarrow \infty$.

Similarly, when $\sum_{i=1}^{n} r_{i}$ is odd, since $q$ is an integer and $s_{j} \geq 2$ for all $j$, we always have $q<\frac{\sum_{i=1}^{n} r_{i}}{2}$. Therefore, the expectation of the corresponding terms satisfying the condition that $\sum_{i=1}^{n} r_{i}$ is odd in the sum of $I\left(q ; s_{1}, \cdots, s_{n}, s_{1,1}, \cdots, s_{q, n}\right)$ will always converge to 0 as $a \rightarrow \infty$.

Case 3 All $s_{j} \geq 2, \sum_{i=1}^{n} r_{i}$ is even and $\frac{\sum_{i=1}^{n} r_{i}}{2}=q$.

The only terms that may not converge to zero are the terms that are not covered in Case 1 and Case 2. This means that the only terms that have nontrivial limits are the terms satisfying the conditions that

$$
\sum_{i=1}^{n} r_{i} \text { is even and } q=\frac{\sum_{i=1}^{n} r_{i}}{2} .
$$

With the condition (iv), we have $\sum_{j=1}^{q} s_{j}=\sum_{i=1}^{n} r_{i}=2 q$. But $s_{j} \geq 2$ for all $j$. Thus it is easy to see this is possible only when

$$
s_{1}=\cdots=s_{q}=2 \quad \text { and } \quad s_{j, i} \in\{0,1,2\}
$$

for all $j \in\{1, \cdots, q\}$ and for all $i \in\{1, \cdots, n\}$. We shall discuss this nontrivial case in 3 steps.

Step 1: The form of $I\left(q ; s_{1}, \cdots, s_{n}, s_{1,1}, \cdots, s_{q, n}\right)$.

For $\ell \in\left\{1, \cdots, \frac{\sum_{i=1}^{n} r_{i}}{2}\right\}$ the factors in $I\left(q ; s_{1}, \cdots, s_{n}, s_{1,1}, \cdots, s_{q, n}\right)$ are either of the form $w_{e_{\ell}}^{2}\left(\delta_{\theta_{e_{\rho}}}\left(A_{i}\right)-H\left(A_{i}\right)\right)\left(\delta_{\theta_{e_{\ell}}}\left(A_{j}\right)-H\left(A_{j}\right)\right)$ for $1 \leq i<j \leq n$ (we call this factor the $(i j)$-mixed term, and if there is no ambiguity to omit the pre-index $(i j)$, we call this kind of factor the mixed term) or of the form $w_{e_{\ell}}^{2}\left(\delta_{\theta_{e_{\ell}}}\left(A_{d}\right)-H\left(A_{d}\right)\right)^{2}$ (which we call the power two term of $A_{d}$ ) for $d \in\{1, \cdots, n\}$.

Step 2: Computation of $C\left(q ; s_{1}, \cdots, s_{n}, s_{1,1}, \cdots, s_{q, n}\right)$.

For each $\ell \in\left\{1, \cdots, \frac{\sum_{i=1}^{n} r_{i}}{2}\right\}$, let

$$
p_{i j}:=\#\left\{(i, j) ; 1 \leq i<j \leq n, s_{\ell, i}=s_{\ell, j}=1\right\} .
$$

Namely, for each pair of $i, j$ such that $1 \leq i<j \leq n, p_{i j}$ is the number of $(i j)$ mixed terms in the product of $I\left(q ; s_{1}, \cdots, s_{n}, s_{1,1}, \cdots, s_{q, n}\right)$. Notice that, in order to obtain a (ij)-mixed term, we need to multiply the form $w_{e_{\ell}}\left(\delta_{\theta_{e_{\ell}}}\left(A_{i}\right)-H\left(A_{i}\right)\right)$ (we call this form the power 1 term of $A_{i}$ and there are $r_{i}$ power 1 terms of $A_{i}$ ) and the form $w_{e_{\ell}}\left(\delta_{\theta_{e_{\ell}}}\left(A_{j}\right)-H\left(A_{j}\right)\right.$ ) (we call this form the power 1 term of $A_{j}$ and there are $r_{j}$ power 1 terms of $\left.A_{i}\right)$. Moreover, there are $p_{i j}$ ! ways to get as many as $p_{i j}(i j)$-mixed terms. Therefore, there are totally $\prod_{1 \leq i<j \leq n} p_{i j} !$ mixed terms in $I\left(q ; s_{1}, \cdots, s_{n}, s_{1,1}, \cdots, s_{q, n}\right)$.

Now for each $d \in\{1, \cdots, n\}$, after picking up $p_{d j}$ power 1 terms of $A_{d}$, there will be $\frac{r_{d}-\sum_{j>d} p_{d j}-\sum_{i<d} p_{i d}}{2}$ power 2 terms of $A_{d}$ left for us to pick up. Thus, for each 
$d \in\{1, \cdots, n\}$, there are $r_{d}$ power 1 terms of $A_{d}$, in which $\sum_{j>d}^{n} p_{d j}+\sum_{i<d} p_{i d}$ of them will be used to construct the mixed terms and $r_{d}-\sum_{j>d}^{n} p_{d j}-\sum_{i<d} p_{i d}$ of them will be used to construct the power 2 terms of $A_{d}$. They have to satisfy the conditions (denoted by $\mathcal{C})$

$$
\left\{\begin{array}{l}
r_{1}-\sum_{j>1} p_{1 j} \text { is even, } \\
r_{\ell}-\sum_{j>\ell} p_{\ell j}-\sum_{i<\ell} p_{i \ell} \text { is even, for } \ell \in\{2, \cdots, n-1\}, \\
r_{n}-\sum_{i<n} p_{i n} \text { is even. }
\end{array}\right.
$$

Thus, for each $d \in\{1, \cdots, n\}$, we can construct the mixed terms and power 2 terms of $A_{d}$ as follows. Since there are $\sum_{j>d}^{n} p_{d j}+\sum_{i<d} p_{i d}$ mixed terms, we choose $\left(p_{1 d}, \cdots, p_{(d-1) d}, p_{d(d+1)}, \cdots, p_{d n}\right)$ (some of them could be 0 ) out of $r_{d}$ and then combine the remaining $r_{d}-\sum_{j>d}^{n} p_{d j}-\sum_{i<d} p_{i d}$ power 1 terms of $A_{d}$ as the power 2 terms of $A_{d}$. Thus, the number of terms from the above steps is

$$
\frac{\left(\begin{array}{c}
r_{d} \\
p_{1 d}, \cdots, p_{(d-1) d}, p_{d(d+1)}, \cdots, p_{d n}
\end{array}\right)\left(\begin{array}{c}
r_{d}-\sum_{j>d}^{n} p_{d j}-\sum_{i<d} p_{i d} \\
2
\end{array}\right)\left(\begin{array}{c}
r_{d}-\sum_{j>d}^{n} p_{d j}-\sum_{i<d} p_{i d}-2 \\
2
\end{array}\right) \cdots\left(\begin{array}{l}
2 \\
2
\end{array}\right)}{\left(\frac{r_{d}-\sum_{j>d}^{n} p_{d j}-\sum_{i<d} p_{i d}}{2}\right) !}
$$

without ordering. After the above steps, all the mixed terms and power 2 terms of $A_{d}$ can be ordered in $\frac{\sum_{l=1}^{n} r_{l}}{2}$ ! ways. Noticing that $p_{d_{1} d_{2}} \in\left\{0,1, \cdots, r_{d_{1}} \wedge r_{d_{2}}\right\}$, the coefficient $C\left(q ; s_{1}, \cdots, s_{n}, s_{1,1}, \cdots, s_{q, n}\right)$ is then

$$
\begin{aligned}
& C\left(q ; s_{1}, \cdots, s_{n}, s_{1,1}, \cdots, s_{q, n}\right) \\
& =\sum_{\substack{p_{i j}=0,1 \leq i<j \leq n \\
(i, j) \in \mathcal{C}}}^{r_{i} \wedge r_{j}} \prod_{d=1}^{n} \frac{\left(\begin{array}{c}
r_{d} \\
p_{1 d}, \cdots, p_{(d-1) d}, p_{d(d+1)}, \cdots, p_{d n}
\end{array}\right)\left(\begin{array}{c}
r_{d}-\sum_{j>d}^{n} p_{d j}-\sum_{i<d} p_{i d} \\
2
\end{array}\right) \cdots\left(\begin{array}{l}
2 \\
2
\end{array}\right)}{\left(\frac{r_{d}-\sum_{j>d}^{n} p_{d j}-\sum_{i<d} p_{i d}}{2}\right) !} \\
& \left(\prod_{1 \leq i<j \leq n} p_{i j} !\right)\left(\frac{\sum_{\ell=1}^{n} r_{\ell}}{2}\right) ! \\
& =\sum_{\substack{p_{i j}=0,1 \leq i<j \leq n \\
(i, j) \in \mathcal{C}}}^{r_{i} \wedge r_{j}} \prod_{d=1}^{n} \frac{\left(\begin{array}{c}
r_{d} \\
p_{1 d}, \cdots, p_{(d-1) d}, p_{d(d+1)}, \cdots, p_{d n}
\end{array}\right)\left(r_{d}-\sum_{j>d}^{n} p_{d j}-\sum_{i<d} p_{i d}\right) !}{\left(\frac{r_{d}-\sum_{j>d}^{n} p_{d j}-\sum_{i<d} p_{i d}}{2}\right) ! 2 \frac{r_{d}-\sum_{j>d}^{n} p_{d j}-\sum_{i<d} p_{i d}}{2}} \\
& \left(\prod_{1 \leq i, j \leq n} p_{i j} !\right)\left(\frac{\sum_{\ell=1}^{n} r_{\ell}}{2} !\right) \\
& =\sum_{\substack{p_{i j}=0,1 \leq i<j \leq n \\
(i, j) \in \mathcal{C}}}^{r_{i} \wedge r_{j}} \frac{r_{1} ! \cdots r_{n} !}{\left(\frac{r_{1}-\sum_{j>1} p_{1 j}}{2}\right) ! \cdots\left(\frac{r_{m}-\sum_{j>m} p_{m j}-\sum_{i<m} p_{i m}}{2}\right) ! \cdots\left(\frac{r_{n}-\sum_{i<n} p_{i n}}{2}\right) !} \\
& \frac{\left(\frac{\sum_{\ell=1}^{n} r_{\ell}}{2}\right) !}{2^{\frac{1}{2} \sum_{\ell=1}^{n} r_{\ell}-\sum_{1 \leq i, j \leq n} p_{i j}}\left(\prod_{1 \leq i<j \leq n} p_{i j} !\right)} .
\end{aligned}
$$


Step 3: Computation of $\mathbb{E}\left[\prod_{i=1}^{n} D_{a}^{r_{i}}\left(A_{i}\right)\right]$.

By Proposition $3.1, I\left(q ; s_{1}, \cdots, s_{n}, s_{1,1}, \cdots, s_{q, n}\right)$ can be rewritten as

$$
\begin{aligned}
& I\left(q ; s_{1}, \cdots, s_{n}, s_{1,1}, \cdots, s_{q, n}\right)= \\
& \mathbb{E}\left[\sum_{1 \leq e_{1}<\cdots<e_{\frac{\sum_{i=1}^{n} r_{i}}{2}}<\infty} \prod_{j=1}^{\frac{\sum_{i=1}^{n} r_{i}}{2}} w_{e_{j}}^{2}\right] \prod_{d=1}^{n}\left(\mathbb{E}\left[\left(\delta_{\theta}\left(A_{d}\right)-H\left(A_{d}\right)\right)^{2}\right]\right) \\
& =\left(\frac{\prod_{1 \leq i<j \leq n}\left(\mathbb{E}\left[\left(\delta_{\theta}\left(A_{i}\right)-H\left(A_{i}\right)\right)\left(\delta_{\theta}\left(A_{j}\right)-H\left(A_{j}\right)\right)\right]\right)^{p_{i j}}}{2^{\frac{\sum_{i=1}^{n} r_{i}}{2}}\left(\frac{\sum_{i=1}^{n} r_{i}}{2}\right) !}\left(\frac{\mathbb{E}\left[v_{1}^{2}\right]}{\mathbb{E}\left[v_{1}\right]}\right)^{\frac{\sum_{i=1}^{n} r_{i}}{2}}+o\left(\left(\frac{\mathbb{E}\left[v_{1}^{2}\right]}{\mathbb{E}\left[v_{1}\right]}\right)^{\frac{\sum_{i=1}^{n} r_{i}}{2}}\right)\right) \\
& \prod_{d=1}^{n}\left(H\left(A_{d}\right)\left(1-H\left(A_{d}\right)\right)\right)^{\frac{r_{d}-\sum_{j>d} p_{i d}}{p_{d j}-\sum_{i<d} p_{i d}}} \\
& \prod_{1 \leq i<j \leq n}\left(-H\left(A_{i}\right) H\left(A_{j}\right)\right)^{p_{i j}} .
\end{aligned}
$$

From (7.56) and (7.58), (7.59), we can compute $\mathbb{E}\left[\prod_{i=1}^{n} D_{a}^{r_{i}}\left(A_{i}\right)\right]$ as follows.

$$
\begin{aligned}
& \mathbb{E}\left[\prod_{i=1}^{n} D_{a}^{r_{i}}\left(A_{i}\right)\right]=\prod_{i=1}^{n}\left[H\left(A_{i}\right)\left(1-H\left(A_{i}\right)\right)\left(\frac{\mathbb{E}\left[v_{1}^{2}\right]}{2 \mathbb{E}\left[v_{1}\right]}+o\left(\frac{\mathbb{E}\left[v_{1}^{2}\right]}{\mathbb{E}\left[v_{1}\right]}\right)\right)\right]^{-\frac{r_{i}}{2}} \\
& \left(\frac{1}{2^{\frac{\sum_{i=1}^{n} r_{i}}{2}}\left(\frac{\sum_{i=1}^{n} r_{i}}{2}\right) !}\left(\frac{\mathbb{E}\left[v_{1}^{2}\right]}{\mathbb{E}\left[v_{1}\right]}\right)^{\frac{\sum_{i=1}^{n} r_{i}}{2}}+o\left(\left(\frac{\mathbb{E}\left[v_{1}^{2}\right]}{\mathbb{E}\left[v_{1}\right]}\right)^{\frac{\sum_{i=1}^{n} r_{i}}{2}}\right)\right) \\
& \sum_{\substack{p_{i j}=0,1 \leq i<j \leq n \\
(i, j) \in \mathcal{C}}}^{r_{i} \wedge r_{j}} \frac{r_{1} ! \cdots r_{n} !}{\left(\frac{r_{1}-\sum_{j>1} p_{1 j}}{2}\right) ! \cdots\left(\frac{r_{m}-\sum_{j>m} p_{m j}-\sum_{i<m} p_{i m}}{2}\right) ! \cdots\left(\frac{r_{n}-\sum_{i<n} p_{i n}}{2}\right) !} \\
& \frac{\left(\frac{\sum_{i=1}^{n} r_{i}}{2}\right) !}{2^{\frac{1}{2} \sum_{\ell=1}^{n} r_{\ell}-\sum_{1 \leq i<j \leq n} p_{i j}}\left(\prod_{1 \leq i<j \leq n} p_{i j} !\right)} \\
& \prod_{d=1}^{n}\left(H\left(A_{d}\right)\left(1-H\left(A_{d}\right)\right)\right)^{\frac{r_{d}-\sum_{j}>d p_{d j}-\sum_{i<d} p_{i d}}{2}} \prod_{1 \leq i<j \leq n}\left(-H\left(A_{i}\right) H\left(A_{j}\right)\right)^{p_{i j}} \\
& =\sum_{\substack{p_{i j}=0,1 \leq i<j \leq n \\
(i, j) \in \mathcal{C}}}^{r_{i} \wedge r_{j}} \frac{r_{1} ! \cdots r_{n} !}{\left(\frac{r_{1}-\sum_{j>1} p_{1 j}}{2}\right) ! \cdots\left(\frac{r_{m}-\sum_{j>m} p_{m j}-\sum_{i<m} p_{i m}}{2}\right) ! \cdots\left(\frac{r_{n}-\sum_{i<n} p_{i n}}{2}\right) !} \\
& \frac{1}{2^{\frac{1}{2} \sum_{\ell=1}^{n} r_{\ell}-\sum_{1 \leq i<j \leq n} p_{i j}}\left(\prod_{1 \leq i<j \leq n} p_{i j} !\right)} \\
& \prod_{1 \leq i<j \leq n}\left(-\sqrt{\frac{H\left(A_{i}\right) H\left(A_{j}\right)}{\left(1-H\left(A_{i}\right)\right)\left(1-H\left(A_{j}\right)\right)}}\right)^{p_{i j}}+o(1)
\end{aligned}
$$




$$
\begin{aligned}
& \stackrel{a \rightarrow \infty}{\rightarrow} \sum_{\substack{p_{i j}=0,1 \leq i<j \leq n \\
(i, j) \in \mathcal{C}}}^{r_{i} \wedge r_{j}} \frac{r_{1} ! \cdots r_{n} !}{\left(\frac{r_{1}-\sum_{j>1} p_{1 j}}{2}\right) ! \cdots\left(\frac{r_{m}-\sum_{j>m} p_{m j}-\sum_{i<m} p_{i m}}{2}\right) ! \cdots\left(\frac{r_{n}-\sum_{i<n} p_{i n}}{2}\right) !} \\
& \frac{1}{2^{\frac{1}{2} \sum_{\ell=1}^{n} r_{\ell}-\sum_{1 \leq i<j \leq n} p_{i j}}\left(\prod_{1 \leq i<j \leq n} p_{i j} !\right)} \\
& \prod_{1 \leq i<j \leq n}\left(-\sqrt{\frac{H\left(A_{i}\right) H\left(A_{j}\right)}{\left(1-H\left(A_{i}\right)\right)\left(1-H\left(A_{j}\right)\right)}}\right)^{p_{i j}},
\end{aligned}
$$

which is equal to $\mathbb{E}\left[\prod_{i=1}^{n} X_{i}^{r_{i}}\right]$, where $X_{1}, \cdots, X_{n}$ are the multi-normal distribution defined in Proposition 7.14. Now, by multinomial expansion we see that for any positive integer $k$ and for any $\left(t_{1}, \cdots, t_{n}\right) \in \mathbb{R}^{d}$,

$$
\begin{aligned}
\mathbb{E}\left[\sum_{i=1}^{n} t_{i} D_{a}\left(A_{i}\right)\right]^{k} & =\sum_{k_{1}+\cdots+k_{n}=k}\left(\begin{array}{c}
k \\
k_{1} ! \cdots k_{n} !
\end{array}\right) \mathbb{E}\left[\prod_{i=1}^{n} t_{i}^{k_{i}} D_{a}^{k_{i}}\left(A_{i}\right)\right] \\
& \sum_{k_{1}+\cdots+k_{n}=k}\left(\begin{array}{c}
k \\
k_{1} ! \cdots k_{n} !
\end{array}\right) \mathbb{E}\left[\prod_{i=1}^{n} t_{i}^{k_{i}} X_{i}^{k_{i}}\right] \\
& =\mathbb{E}\left[\sum_{i=1}^{n} t_{i} X_{i}\right]^{k} .
\end{aligned}
$$

By the method of moments (see e.g. Billingsley (1995, Theorem 30.2)) it follows that

$$
\sum_{i=1}^{n} t_{i} D_{a}\left(A_{i}\right) \stackrel{d}{\rightarrow} \sum_{i=1}^{n} t_{i} X_{i} \quad \text { as } a \rightarrow \infty .
$$

Part (i) of Theorem 4.4 follows then from the Cramér-Wold theorem (e.g. Billingsley (1995, Theorem 29.4)).

Now, we prove the part (ii) of this theorem by proving the weak convergence of finite dimensional distributions and by verifying a tightness condition. The finite dimensional weak convergence of $Q_{H, a}$ can be shown directly by part (i), i.e. for any finite measurable sets $A_{1}, \cdots, A_{n}$ in $\mathcal{X}^{d}$, we have

$$
\left(Q_{H, a}\left(A_{1}\right), \cdots, Q_{H, a}\left(A_{n}\right)\right) \stackrel{d}{\rightarrow}\left(B_{H}^{o}\left(A_{1}\right), \cdots, B_{H}^{o}\left(A_{n}\right)\right) .
$$

By Theorem 2 of Bickel and Wichura (1971), to show (4.7) we only need to check the tightness condition, i.e, inequality (3) of Bickel and Wichura (1971), with $\gamma_{1}=\gamma_{2}=2$, $\beta_{1}=\beta_{2}=1$ and $\mu=2 H$. Obviously, $\mu$ is finite and nonatomic. For every pair of Borel sets $A$ and $B$ in $\mathcal{B}\left(\mathbb{R}^{d}\right)$, by the proof of part (i) of this theorem and the Isserlis' theorem Isserlis (1918) we have

$$
\begin{aligned}
& \mathbb{E}\left[\left|Q_{H, a}(A)\right|^{2}\left|Q_{H, a}(B)\right|^{2}\right] \\
& =[H(A)(1-H(A))][H(B)(1-H(B))] \mathbb{E}\left[D_{a}^{2}(A) D_{a}^{2}(B)\right]
\end{aligned}
$$




$$
\begin{aligned}
& =[H(A)(1-H(A))][H(B)(1-H(B))]\left(1+2 \frac{H(A) H(B)}{(1-H(A))(1-H(B))}+o(1)\right) \\
& =3 H(A)^{2} H(B)^{2}-H(A)^{2} H(B)-H(A) H(B)^{2}+H(A) H(B)+o(1) \\
& \leq \mu(A) \mu(B) .
\end{aligned}
$$

The last inequality is due to the fact that $H(\cdot) \in(0,1)$ and thus $H(\cdot)^{2} \leq H(\cdot)$. Therefore, the tightness condition on $D\left(\mathbb{R}^{d}\right)$ is verified.

\subsection{Proof of Theorem 4.15}

Proof of Theorem 4.15. Once we have Proposition 3.2-3.4, the proofs of part (i) of this theorem for the various processes except the generalized Dirichlet process follow from a similar argument to that in the proof of part (i) of Theorem 4.4. So, we shall omit the details.

When $P \sim \operatorname{GDP}(a, r, H)$, We need the following result about the variance of $P$ from Lijoi et al. (2005a):

$$
\operatorname{Var}[P(A)]=H(A)(1-H(A)) \mathcal{I}_{a, r},
$$

where $\mathcal{I}_{a, r}$ is given by

$$
\begin{aligned}
\mathcal{I}_{a, r} & =a(r !)^{a} \sum_{k=1}^{r} \int_{0}^{\infty} \frac{x}{(k+x)^{2} \prod_{j=1}^{r}(j+x)^{a}} d x \\
& =\frac{a(r !)^{a} \Gamma(r a)}{r^{r a} \Gamma(r a+2)} \sum_{j=1}^{r} F_{D}^{(r-1)}\left(r a, \mathbf{a}_{\mathbf{k}}^{*} ; r a+2 ; \frac{1}{r} \mathbf{J}_{\mathbf{r}-\mathbf{1}}\right) .
\end{aligned}
$$

Here $\mathbf{a}_{k}^{*}=(a, \cdots, a+2, \cdots, a)^{T}$ is a $r-1$ dimensional vector where the $k$-th element is $a+2$ and all other elements are equal to $a ; \mathbf{J}_{r-1}=(1, \cdots, r-1)^{T}$; and $F_{D}^{(r-1)}$ is the fourth Lauricella multiple hypergeometric function (see e.g. Exton (1976) for more details).

Letting $x=\frac{t}{a}$ we have

$$
\begin{aligned}
\mathcal{I}_{a, r} & =a \sum_{k=1}^{r} \frac{1}{k^{2}} \int_{0}^{\infty} \frac{x}{\left(1+\frac{x}{k}\right)^{2} \prod_{j=1}^{r}\left(1+\frac{x}{j}\right)^{a}} d x \\
& =a \sum_{k=1}^{r} \frac{1}{a k^{2}} \int_{0}^{\infty} \frac{t}{a\left(1+\frac{t}{a k}\right)^{2} \prod_{j=1}^{r}\left(1+\frac{t}{a j}\right)^{a}} d t .
\end{aligned}
$$

When $a$ is large, we can approximate $\mathcal{I}_{a, r}$ by

$$
\mathcal{I}_{a, r}=\frac{1}{a} \sum_{k=1}^{r} \frac{1}{k^{2}} \int_{0}^{\infty} t e^{-\left(\sum_{j=1}^{r} \frac{1}{j}\right) t} d t+o\left(\frac{1}{a}\right)
$$




$$
=\frac{\sum_{k=1}^{r}\left(\frac{1}{k}\right)^{2}}{\left(\sum_{j=1}^{r} \frac{1}{j}\right)^{2} a}+o\left(\frac{1}{a}\right) .
$$

Denote

$$
c=\frac{\left(\sum_{j=1}^{r} \frac{1}{j}\right)^{2}}{\sum_{k=1}^{r}\left(\frac{1}{k}\right)^{2}} .
$$

Then

$$
D_{a}(\cdot) \approx \frac{1}{\sqrt{\frac{H(\cdot)(1-H(\cdot))}{c a}}}(P(\cdot)-H(\cdot)),
$$

where $\approx$ means that the two sides converge to the same distribution as $a \rightarrow \infty$. We shall prove the result for $n=3$ and the case for general $n$ can be handled in a similar way.

By the integral representation of the confluent form of the fourth Lauricella hypergeometric function (see e.g. formula (1.4.3.9) in Exton (1976)), the joint probability density of $P\left(A_{1}\right), P\left(A_{2}\right)$ admits the following form:

$$
\begin{aligned}
\rho\left(x_{1}, x_{2}\right)= & \frac{\Gamma(r a)}{\Gamma\left(r a H_{1}\right) \Gamma\left(r a H_{2}\right) \Gamma\left(r a H_{3}\right)} \frac{(r !)}{r^{r a} \Gamma(r a)} \frac{\Gamma\left(r a H_{1}\right) \Gamma\left(r a H_{2}\right) \Gamma\left(r a H_{3}\right)}{\left[\Gamma\left(a H_{1}\right) \Gamma\left(a H_{2}\right) \Gamma\left(a H_{3}\right)\right]^{r}} \\
& \times x_{1}^{2 a H_{1}-1} x_{2}^{2 a H_{2}-1}\left(1-x_{1}-x_{2}\right)^{2 a H_{3}-1} \\
& \times \int_{0}^{\infty} \int_{0 \leq u_{1}^{(1)}+\cdots+u_{r-1}^{(1)} \leq 1} \int_{0 \leq u_{1}^{(2)}+\cdots+u_{r-1}^{(2)} \leq 1} \int_{0 \leq u_{1}^{(3)}+\cdots+u_{r-1}^{(3)} \leq 1} \xi^{r a-1} \\
& \times \exp \left\{-\xi+\xi\left(\frac{\sum_{k=1}^{r-1} k u_{k}^{(1)} x_{1}+\sum_{k=1}^{r-1} k u_{k}^{(2)} x_{2}+\sum_{k=1}^{r-1} k u_{k}^{(3)}\left(1-x_{1}-x_{2}\right)}{r}\right)\right\} \\
& \times \prod_{i=1}^{3}\left[u_{1}^{i} \cdots u_{r-1}^{(i)}\left(1-u_{1}^{i}-\cdots-u_{r-1}^{(i)}\right)\right]^{a H_{i}-1} d u_{1}^{(1)} \cdots d u_{r-1}^{(1)} \\
& d u_{1}^{(2)} \cdots d u_{r-1}^{(2)} d u_{1}^{(3)} \cdots d u_{r-1}^{(3)} d \xi
\end{aligned}
$$

where $H_{i}=H\left(A_{i}\right)$ for $i=1,2,3$. Using the expression of (7.64) and the above density form (7.65), we can obtain the probability density function of $D\left(A_{1}\right), D\left(A_{2}\right)$ as follows:

$$
f\left(y_{1}, y_{2}\right)=\mathcal{J}_{1} \times f_{1}\left(y_{1}, y_{2}\right),
$$

where

$$
\begin{aligned}
& \mathcal{J}_{1}=\frac{\sqrt{H_{1}\left(1-H_{1}\right) H_{2}\left(1-H_{2}\right)} \Gamma(r a)}{c a \Gamma\left(r a H_{1}\right) \Gamma\left(r a H_{2}\right) \Gamma\left(r a H_{3}\right)}\left(\sqrt{\frac{H_{1}\left(1-H_{1}\right)}{c a}} y_{1}+H_{1}\right)^{r a H_{1}-1} \\
& \times\left(\sqrt{\frac{H_{2}\left(1-H_{2}\right)}{c a}} y_{2}+H_{2}\right)^{r a H_{2}-1}\left(H_{3}-\frac{\sqrt{H_{1}\left(1-H_{1}\right)} y_{1}+\sqrt{H_{2}\left(1-H_{2}\right)} y_{2}}{\sqrt{c a}}\right)^{r a H_{3}-1}
\end{aligned}
$$


and

$$
\begin{aligned}
f_{1}\left(y_{1}, y_{2}\right)= & \int_{0}^{\infty} \int \cdots \int_{0 \leq u_{1}^{(1)}+\cdots+u_{r-1}^{(1)} \leq 1} \int \cdots \int_{0 \leq u_{1}^{(2)}+\cdots+u_{r-1}^{(2)} \leq 1} \\
& \int \cdots \int_{0 \leq u_{1}^{(3)}+\cdots+u_{r-1}^{(3)} \leq 1} \prod_{i=1}^{3}\left[u_{1}^{i} \cdots u_{r-1}^{(i)}\left(1-u_{1}^{i}-\cdots-u_{r-1}^{(i)}\right)\right]^{a H_{i}-1} \xi^{r a-1} \\
& \times \exp \left\{-\xi+\frac{\xi}{r}\left[\sum_{k=1}^{r-1} k u_{k}^{(1)}\left(\frac{\sqrt{H_{1}\left(1-H_{1}\right)} y_{1}}{\sqrt{c a}}+H_{1}\right)\right.\right. \\
& +\sum_{k=1}^{r-1} k u_{k}^{(2)}\left(\frac{\sqrt{H_{2}\left(1-H_{2}\right)} y_{2}}{\sqrt{c a}+H_{2}}\right) \\
& \left.\left.+\sum_{k=1}^{r-1} k u_{k}^{(3)}\left(H_{3}-\frac{\sqrt{H_{1}\left(1-H_{1}\right)} y_{1}+\sqrt{H_{2}\left(1-H_{2}\right)} y_{2}}{\sqrt{c a}}\right)\right]\right\} \\
& d u_{1}^{(1)} \cdots d u_{r-1}^{(1)} d u_{1}^{(2)} \cdots d u_{r-1}^{(2)} d u_{1}^{(3)} \cdots d u_{r-1}^{(3)} d \xi .
\end{aligned}
$$

To determine if the density of $f\left(y_{1}, y_{2}\right)$ has a limit or not and if yes, to find the limiting density, we shall find the limits of $\mathcal{J}_{1}$ and $f_{1}\left(y_{1}, y_{2}\right)$ separately.

\section{Step 1: Limit of $\mathcal{J}_{1}$.}

By Stirling's formula $\Gamma(z)=\sqrt{2 \pi} z^{z-\frac{1}{2}} e^{-z}\left(1+o\left(\frac{1}{z}\right)\right)$ when $z$ is large, we have the following approximation:

$$
\begin{aligned}
\mathcal{J}_{1}= & \frac{r a}{c a 2 \pi} \sqrt{\frac{\left(1-H_{1}\right)\left(1-H_{2}\right)}{H_{3}}}\left(\sqrt{\frac{\left(1-H_{1}\right)}{c a H_{1}}} y_{1}+1\right)^{r a H_{1}-1}\left(\sqrt{\frac{\left(1-H_{2}\right)}{c a H_{2}}} y_{2}+1\right)^{r a H_{2}-1} \\
& \times\left(1-\frac{\sqrt{H_{1}\left(1-H_{1}\right)} y_{1}+\sqrt{H_{2}\left(1-H_{2}\right)} y_{2}}{H_{3} \sqrt{c a}}\right)^{r a H_{3}-1}\left(1+o\left(\frac{1}{a}\right)\right) \\
= & \frac{r}{c 2 \pi} \sqrt{\frac{\left(1-H_{1}\right)\left(1-H_{2}\right)}{H_{3}}}\left(1+o\left(\frac{1}{a}\right)\right) \exp \left\{r a \left[H_{1} \log \left(\sqrt{\frac{\left(1-H_{1}\right)}{c a H_{1}}} y_{1}+1\right)\right.\right. \\
& +H_{2} \log \left(\sqrt{\frac{\left(1-H_{2}\right)}{c a H_{2}}} y_{2}+1\right) \\
& \left.\left.+H_{3} \log \left(1-\frac{\sqrt{H_{1}\left(1-H_{1}\right)} y_{1}+\sqrt{H_{2}\left(1-H_{2}\right)} y_{2}}{H_{3} \sqrt{c a}}\right)\right]\right\} .
\end{aligned}
$$

An application of $\log \left(1+\frac{1}{\sqrt{z}}\right)=\frac{1}{\sqrt{z}}-\frac{1}{2 z}+o\left(\frac{1}{z}\right)$ for large $z$ yields

$$
\mathcal{J}_{1}=\frac{r}{c} \frac{\sqrt{\left(1-H_{1}\right)\left(1-H_{2}\right)}}{2 \pi \sqrt{H_{3}}}
$$




$$
\times \exp \left\{-\frac{r}{c} \frac{y_{1}^{2}+y_{2}^{2}+2 y_{1} y_{2} \sqrt{\frac{H_{1} H_{2}}{\left(1-H_{1}\right)\left(1-H_{2}\right)}}}{2 \frac{H_{3}}{\left(1-H_{1}\right)\left(1-H_{2}\right)}}\right\}\left(1+o\left(\frac{1}{a}\right)\right) .
$$

Step 2: The limit of $f_{1}\left(y_{1}, y_{2}\right)$. This is much more complicated. We first obtain the leading terms of $f_{1}$ as $a \rightarrow \infty$.

To make the presentation clear, denote the integrating variables by

$$
\mathbf{z}=\left(u_{1}^{(1)}, \cdots, u_{r-1}^{(1)}, u_{1}^{(2)}, \cdots, u_{r-1}^{(2)}, u_{1}^{(3)}, \cdots, u_{r-1}^{(3)}, \xi\right)^{T}
$$

and denote

$$
\begin{aligned}
g\left(u_{1}^{(1)}, \cdots, u_{r-1}^{(1)}, u_{1}^{(2)}, \cdots, u_{r-1}^{(2)}, u_{1}^{(3)}, \cdots, d u_{r-1}^{(3)}, \xi\right) \\
:=\sum_{i=1}^{3}\left(a H_{i}-1\right) \log \left[u_{1}^{(i)} \cdots u_{r-1}^{(i)}\left(1-u_{1}^{(i)}-\cdots-u_{r-1}^{(i)}\right)\right]+(r a-1) \log (\xi) \\
-\xi+\frac{\xi}{r}\left[\sum_{k=1}^{r-1} k u_{k}^{(1)}\left(\frac{\sqrt{H_{1}\left(1-H_{1}\right)} y_{1}}{\sqrt{c a}}+H_{1}\right)+\sum_{k=1}^{r-1} k u_{k}^{(2)}\left(\frac{\sqrt{H_{2}\left(1-H_{2}\right)} y_{2}}{\sqrt{c a}}+H_{2}\right)\right. \\
\left.\quad+\sum_{k=1}^{r-1} k u_{k}^{(3)}\left(H_{3}-\frac{\sqrt{H_{1}\left(1-H_{1}\right)} y_{1}+\sqrt{H_{2}\left(1-H_{2}\right)} y_{2}}{\sqrt{c a}}\right)\right]
\end{aligned}
$$

This function $g$ attains its maximum at its critical point

$$
\mathbf{z}_{\mathbf{0}}=\left(u_{1,0}^{(1)}, \cdots, u_{r-1,0}^{(1)}, u_{1,0}^{(2)}, \cdots, u_{r-1,0}^{(2)}, u_{1,0}^{(3)}, \cdots, u_{r-1,0}^{(3)}, \xi_{0}\right)^{T}
$$

where

$$
\left\{\begin{array}{l}
u_{k, 0}^{(i)}=\frac{1}{(r-k)\left(\sum_{j=1}^{r} \frac{1}{j}\right)}, \quad k=1, \cdots, r-1, i=1,2,3 \\
\xi_{0}=(r a-1)\left(\sum_{j=1}^{r} \frac{1}{j}\right) .
\end{array}\right.
$$

By an elementary calculation we have

$$
g^{\prime}\left(\mathbf{z}_{\mathbf{0}}\right)=\left(\begin{array}{c}
{\left[\left(\sum_{j=1}^{r} \frac{1}{j}\right)\left(1-\frac{H_{1}}{r}\right)+\left(\sum_{j=1}^{r} \frac{1}{j}\right)\left(a-\frac{1}{r}\right) \frac{\sqrt{H_{1}\left(1-H_{1}\right)} y_{1}}{\sqrt{c a}}\right] \mathbf{J}_{r-1}} \\
{\left[\left(\sum_{j=1}^{r} \frac{1}{j}\right)\left(1-\frac{H_{2}}{r}\right)+\left(\sum_{j=1}^{r} \frac{1}{j}\right)\left(a-\frac{1}{r}\right) \frac{\sqrt{H_{2}\left(1-H_{2}\right)} y_{2}}{\sqrt{c a}}\right] \mathbf{J}_{r-1}} \\
{\left[\left(\sum_{j=1}^{r} \frac{1}{j}\right)\left(1-\frac{H_{1}}{r}\right)-\left(\sum_{j=1}^{r} \frac{1}{j}\right)\left(a-\frac{1}{r}\right) \frac{\sqrt{H_{1}\left(1-H_{1}\right)} y_{1}+\sqrt{H_{2}\left(1-H_{2}\right)} y_{2}}{\sqrt{c a}}\right] \mathbf{J}_{r-1}} \\
0
\end{array}\right),
$$


where $\mathbf{J}_{r-1}=(1, \cdots, r-1)^{T}$ and

$$
g^{\prime \prime}\left(\mathbf{z}_{\mathbf{0}}\right)=\left(\begin{array}{cccc}
M_{1} & & & N_{1} \\
& M_{2} & & N_{2} \\
& & M_{3} & N_{3} \\
N_{1}^{T} & N_{2}^{T} & N_{3}^{T} & -\frac{1}{(r a-1)\left(\sum_{k=1}^{r-1} \frac{1}{k}\right)^{2}}
\end{array}\right),
$$

where the empty entries should be filled with a $(r-1) \times(r-1)$ dimensional zero matrix and where for $i=1,2,3, M_{i}$ is a $(r-1) \times(r-1)$ matrix given by

$$
M_{i}=-\left(a H_{i}-1\right)\left(\sum_{k=1}^{r-1} \frac{1}{k}\right)^{2}\left(\begin{array}{cccc}
{\left[(r-1)^{2}+r^{2}\right]} & r^{2} & \cdots & r^{2} \\
r^{2} & {\left[(r-2)^{2}+r^{2}\right]} & \cdots & r^{2} \\
\vdots & \vdots & \ddots & \vdots \\
r^{2} & r^{2} & \cdots & {\left[(1)^{2}+r^{2}\right]}
\end{array}\right) \text {, }
$$

and $N_{i}$ is a $(r-1)$ column vector given by

$$
N_{i}=\left(\begin{array}{c}
\frac{1\left(\frac{\sqrt{H_{i}\left(1-H_{i}\right)} y_{i}}{\sqrt{c a}}+H_{i}\right)}{r} \\
\frac{2\left(\frac{\sqrt{H_{i}\left(1-H_{i}\right)} y_{i}}{\sqrt{c a}}+H_{i}\right)}{r} \\
\vdots \\
\frac{(r-1)\left(\frac{\sqrt{H_{i}\left(1-H_{i}\right)} y_{i}}{\sqrt{c a}}+H_{i}\right)}{r}
\end{array}\right) .
$$

With these notations we have when $a$ is large

$$
\begin{aligned}
f_{1}\left(y_{1}, y_{2}\right)= & \frac{(r !)}{r^{r a} \Gamma(r a)} \frac{\Gamma\left(r a H_{1}\right) \Gamma\left(r a H_{2}\right) \Gamma\left(r a H_{3}\right)}{\left[\Gamma\left(a H_{1}\right) \Gamma\left(a H_{2}\right) \Gamma\left(a H_{3}\right)\right]^{r}} \int_{0}^{\infty} \int \cdots \int_{0 \leq u_{1}^{(1)}+\cdots+u_{r-1}^{(1)} \leq 1} \\
& \int \cdots \int_{0 \leq u_{1}^{(2)}+\cdots+u_{r-1}^{(2)} \leq 1} \int \cdots \int_{0 \leq u_{1}^{(3)}+\cdots+u_{r-1}^{(3)} \leq 1} e^{g(\mathbf{z})} d \mathbf{z} \\
= & \frac{(r !)\left(a H_{1}\right)^{\frac{r-1}{2}}\left(a H_{2}\right)^{\frac{r-1}{2}}\left(a H_{3}\right)^{\frac{r-1}{2}}}{r^{\frac{3}{2}}(\sqrt{2 \pi})^{3 r-2} e^{-r a}(r a)^{r a-\frac{1}{2}}} \int_{0}^{\infty} \int \cdots \int_{0 \leq u_{1}^{(1)}+\cdots+u_{r-1}^{(1)} \leq 1} \exp \left\{g\left(\mathbf{z}_{\mathbf{0}}\right)\right. \\
& \left.\int \cdots \int_{0 \leq u_{1}^{(2)}+\cdots+u_{r-1}^{(2)} \leq 1} \int \cdots \int_{0 \leq u_{1}^{(3)}+\cdots+u_{r-1}^{(3)} \leq 1} \operatorname{er}\left(\frac{1}{a}\right)\right) d \mathbf{z} . \\
& \left.+g^{\prime}\left(\mathbf{z}_{\mathbf{0}}\right)^{T}\left(\mathbf{z}-\mathbf{z}_{\mathbf{0}}\right)+\frac{1}{2}\left(\mathbf{z}-\mathbf{z}_{\mathbf{0}}\right)^{T} g^{\prime \prime}\left(\mathbf{z}_{\mathbf{0}}\right)\left(\mathbf{z}-\mathbf{z}_{\mathbf{0}}\right)\right\}(1+o(1)
\end{aligned}
$$

\section{Step 3: Evaluation of the leading term of $f_{1}\left(y_{1}, y_{2}\right)$.}

In order to evaluate the integral of (7.72), we use the change of variables

$$
u_{k}^{(i)}-u_{k, 0}^{(i)}=\frac{t_{k}^{(i)}}{\sqrt{a H_{i}} \sqrt{(r-k)^{2}+r^{2}}\left(\sum_{k=1}^{r-1} \frac{1}{k}\right)},
$$


for $k=1, \cdots, r-1, i=1,2,3$, and

$$
\xi-\xi_{0}=\sqrt{r a-1}\left(\sum_{k=1}^{r-1} \frac{1}{k}\right) s .
$$

Thus, we have

$$
\begin{aligned}
f_{1}\left(y_{1}, y_{2}\right)= & \frac{(r !)\left(a H_{1}\right)^{\frac{r-1}{2}}\left(a H_{2}\right)^{\frac{r-1}{2}}\left(a H_{3}\right)^{\frac{r-1}{2}}}{r^{\frac{3}{2}}(\sqrt{2 \pi})^{3 r-2} e^{-r a}(r a)^{r a-\frac{1}{2}}} \sqrt{r a-1}\left(\sum_{k=1}^{r} \frac{1}{k}\right) \\
& \times \prod_{i=1}^{3} \prod_{k=1}^{r-1} \frac{1}{\sqrt{a H_{i}} \sqrt{(r-k)^{2}+r^{2}}\left(\sum_{k=1}^{r} \frac{1}{k}\right)} \\
& \times \int_{-\infty}^{\infty} \cdots \int_{-\infty}^{\infty} \exp \left\{g\left(\mathbf{z}_{\mathbf{0}}\right)+\mathbf{b}^{T} \mathbf{t}-\frac{1}{2} \mathbf{t}^{T} A \mathbf{t}\right\}\left(1+o\left(\frac{1}{a}\right)\right) d \mathbf{t} \\
= & \frac{(r !)^{3}\left(\sum_{k=1}^{r} \frac{1}{k}\right)^{3}}{r^{\frac{3}{2}} \prod_{k=1}^{r-1}\left(\sqrt{(r-k)^{2}+r^{2}}\right)^{3}}(\operatorname{det} A)^{-\frac{1}{2}} \\
& \quad \times \exp \left\{\frac{1}{2} \mathbf{b}^{T} A^{-1} \mathbf{b}\right\}\left(1+o\left(\frac{1}{a}\right)\right),
\end{aligned}
$$

where $\mathbf{t}=\left(t_{1}^{(1)}, \cdots, t_{r-1}^{(2)}, t_{1}^{(2)}, \cdots, t_{r-1}^{(2)}, t_{1}^{(3)}, \cdots, t_{r-1}^{(3)}, s\right)^{T}$, and where a direct calculation from $g^{\prime}\left(\mathbf{z}_{\mathbf{0}}\right)$ gives

$$
\mathbf{b}=\left(\begin{array}{c}
-\frac{\sqrt{r} \sqrt{1-H_{1}} y_{1}}{\sqrt{c}} B \\
-\frac{\sqrt{r} \sqrt{1-H_{2}} y_{2}}{\sqrt{c}} B \\
-\frac{\sqrt{r}\left(\sqrt{H_{1}\left(1-H_{1}\right)} y_{1}+\sqrt{H_{2}\left(1-H_{2}\right)} y_{2}\right)}{\sqrt{c} \sqrt{H_{3}}} B \\
0
\end{array}\right) .
$$

The matrix $A$ in (7.73) can be found directly from $g^{\prime \prime}\left(\mathbf{z}_{\mathbf{0}}\right)$ and will be given below when we study it. The above last identity (7.73) follows from the fact that

$$
e^{g\left(\mathbf{z}_{\mathbf{0}}\right)}=\frac{\left[(r a-1)\left(\sum_{k=1}^{r} \frac{1}{k}\right)\right]^{r a-1}}{\left[r !\left(\sum_{k=1}^{r} \frac{1}{k}\right)^{r}\right]^{a-3}} e^{-(r a-1)}
$$

and the multivariate Gaussian integral formula. By a simple algebra we can write

$$
A=\left(\begin{array}{cccc}
A_{0} & & & B_{1} \\
& A_{0} & & B_{2} \\
& & A_{0} & B_{3} \\
B_{1}^{T} & B_{2}^{T} & B_{3}^{T} & 1
\end{array}\right),
$$

where $A_{0}$ is a $(r-1) \times(r-1)$ matrix whose entries are

$$
\left[A_{0}\right]_{i j}= \begin{cases}1 & \text { if } i=j \\ \frac{r^{2}}{\sqrt{(r-i)^{2}+r^{2}} \sqrt{(r-j)^{2}+r^{2}}} & \text { if } i \neq j\end{cases}
$$


and for $i=1,2,3$

$$
B_{i}=-\left(\begin{array}{c}
\frac{1 \sqrt{r a-1}\left(\frac{\sqrt{H_{i}\left(1-H_{i}\right)} y_{i}}{\sqrt{c a}}+H_{i}\right)}{r \sqrt{a H_{i}} \sqrt{(r-1)^{2}+r^{2}}} \\
\frac{2 \sqrt{r a-1}\left(\frac{\sqrt{H_{i}\left(1-H_{i}\right)} y_{i}}{\sqrt{c a}}+H_{i}\right)}{r \sqrt{a H_{i}} \sqrt{(r-2)^{2}+r^{2}}} \\
\vdots \\
\frac{(r-1) \sqrt{r a-1}\left(\frac{\sqrt{H_{i}\left(1-H_{i}\right)} y_{i}}{\sqrt{c a}}+H_{i}\right)}{r \sqrt{a H_{i}} \sqrt{(1)^{2}+r^{2}}}
\end{array}\right)=-\left(\begin{array}{c}
\frac{1 \sqrt{H_{1}}}{\sqrt{r} \sqrt{(r-1)^{2}+r^{2}}}\left(1+o\left(\frac{1}{\sqrt{a}}\right)\right) \\
\frac{2 \sqrt{H_{1}}}{\sqrt{r} \sqrt{(r-2)^{2}+r^{2}}}\left(1+o\left(\frac{1}{\sqrt{a}}\right)\right) \\
\vdots \\
\frac{(r-1) \sqrt{H_{1}}}{\sqrt{r} \sqrt{(1)^{2}+r^{2}}}\left(1+o\left(\frac{1}{\sqrt{a}}\right)\right)
\end{array}\right) .
$$

Step 4: The inverse and the determinant of $A$

We need to find $A^{-1}$. First we find $A_{0}^{-1}$. From the expression of $A_{0}$ we can write

$$
A_{0}=D+\mathbf{v} \mathbf{v}^{T}=D^{\frac{1}{2}}\left(I+D^{-\frac{1}{2}} \mathbf{v v}^{T} D^{-\frac{1}{2}}\right) D^{\frac{1}{2}}
$$

where

$$
\mathbf{v}=\left(\begin{array}{c}
\frac{r}{\sqrt{(r-1)^{2}+r^{2}}} \\
\frac{r}{\sqrt{(r-2)^{2}+r^{2}}} \\
\vdots \\
\frac{r}{\sqrt{(1)^{2}+r^{2}}}
\end{array}\right)
$$

and

$$
D=\left(\begin{array}{cccc}
\frac{(r-1)^{2}}{(r-1)^{2}+r^{2}} & 0 & \cdots & 0 \\
0 & \frac{(r-2)^{2}}{(r-2)^{2}+r^{2}} & \cdots & 0 \\
\vdots & \vdots & \ddots & \vdots \\
0 & 0 & \cdots & \frac{1^{2}}{1^{2}+r^{2}}
\end{array}\right)
$$

Hence, the determinant of $A_{0}$ is given by

$$
\begin{aligned}
\operatorname{det}\left(A_{0}\right) & =\operatorname{det}\left(D^{\frac{1}{2}}\right) \operatorname{det}\left(I+D^{-\frac{1}{2}} \mathbf{v} \mathbf{v}^{T} D^{-\frac{1}{2}}\right) \operatorname{det}\left(D^{\frac{1}{2}}\right) \\
& =\operatorname{det}\left(D^{\frac{1}{2}}\right)\left(1+\left(D^{-\frac{1}{2}} \mathbf{v}\right)^{T}\left(D^{-\frac{1}{2}} \mathbf{v}\right)\right) \operatorname{det}\left(D^{\frac{1}{2}}\right) \\
& =\left(\frac{(r-1) !}{\prod_{k=1}^{r-1} \sqrt{(r-k)^{2}+r^{2}}}\right)^{2}\left(1+\sum_{k=1}^{r-1} \frac{r^{2}}{(r-k)^{2}}\right) \\
& =\frac{\sum_{k=1}^{r}\left(\frac{r !}{k}\right)^{2}}{\prod_{k=1}^{r-1}\left(\sqrt{(r-k)^{2}+r^{2}}\right)^{2}} .
\end{aligned}
$$

Now, by the Sherman-Morrison formula we have

$$
A_{0}^{-1}=\left(D+f f^{T}\right)^{-1}=D^{-1}-\frac{1}{1+f^{T} D^{-1} f} D^{-1} f f^{T} D^{-1} .
$$


As a result we obtain

$$
\left[A_{0}^{-1}\right]_{i j}= \begin{cases}-\frac{1}{\sum_{k=1}^{r}\left(\frac{1}{k}\right)^{2}} \frac{\sqrt{(r-i)^{2}+r^{2}} \sqrt{(r-j)^{2}+r^{2}}}{(r-i)^{2}(r-j)^{2}} & \text { if } i \neq j \\ \frac{\left[\sum_{k=1}^{r}\left(\frac{1}{k}\right)^{2}-\frac{1}{(r-i)^{2}}\right]}{\sum_{k=1}^{r}\left(\frac{1}{k}\right)^{2}} \frac{\left[(r-i)^{2}+r^{2}\right]}{(r-i)^{2}} & \text { if } i=j .\end{cases}
$$

The determinant of $A$ can be computed as follows.

$$
\begin{aligned}
\operatorname{det}(A) & =\left(\operatorname{det} A_{0}\right)^{3}\left(1-B_{1}^{T} A_{0}^{-1} B_{1}-B_{2}^{T} A_{0}^{-1} B_{2}-B_{3}^{T} A_{0}^{-1} B_{3}\right) \\
& =\left(\operatorname{det} A_{0}\right)^{3}\left(1-B^{T} A_{0}^{-1} B\right),
\end{aligned}
$$

where

$$
B=-\left(\begin{array}{c}
\frac{1}{\sqrt{r} \sqrt{(r-1)^{2}+r^{2}}}\left(1+o\left(\frac{1}{\sqrt{a}}\right)\right) \\
\frac{2}{\sqrt{r} \sqrt{(r-2)^{2}+r^{2}}}\left(1+o\left(\frac{1}{\sqrt{a}}\right)\right) \\
\vdots \\
\frac{(r-1)}{\sqrt{r} \sqrt{(1)^{2}+r^{2}}}\left(1+o\left(\frac{1}{\sqrt{a}}\right)\right)
\end{array}\right) .
$$

From the relation (7.76) of expressing $\operatorname{det}(A)$ by $\operatorname{det}\left(A_{0}\right)$ and $B$ we have

$$
\operatorname{det}(A)=\left(\operatorname{det} A_{0}\right)^{3}\left(1-B^{T} A_{0}^{-1} B\right)=\frac{\left(\sum_{k=1}^{r} \frac{r !}{k}\right)^{2}\left(\sum_{k=1}^{r}\left(\frac{r !}{k}\right)^{2}\right)^{2}}{r \prod_{k=1}^{r-1}\left(\sqrt{(r-k)^{2}+r^{2}}\right)^{6}} .
$$

Moreover, by the block Gaussian elimination method, we find $A^{-1}$ as

$$
\left(\begin{array}{cccc}
A_{0}^{-1}\left(I_{0}+\frac{B_{1} B_{1}^{T} A_{0}^{-1}}{m}\right) & \frac{A_{0}^{-1} B_{1} B_{2}^{T} A_{0}^{-1}}{m} & \frac{A_{0}^{-1} B_{1} B_{3}^{T} A_{0}^{-1}}{m} & -\frac{A_{0}^{-1} B_{1}}{m} \\
\frac{A_{0}^{-1} B_{2} B_{1}^{T} A_{0}^{-1}}{m} & A_{0}^{-1}\left(I_{0}+\frac{B_{2} B_{2}^{T} A_{0}^{-1}}{m}\right) & \frac{A_{0}^{-1} B_{2} B_{3}^{T} A_{0}^{-1}}{m} & -\frac{A_{0}^{-1} B_{2}}{m} \\
\frac{A_{0}^{-1} B_{3} B_{1}^{T} A_{0}^{-1}}{m} & \frac{A_{0}^{-1} B_{3} B_{2}^{T} A_{0}^{-1}}{m} & A_{0}^{-1}\left(I_{0}+\frac{B_{3} B_{3}^{T} A_{0}^{-1}}{m}\right) & \left.-\frac{A_{0}^{-1} B_{3}}{m}\right) \\
-\frac{B_{1}^{T} A_{0}^{-1}}{m} & -\frac{B_{2}^{T} A_{0}^{-1}}{m} & -\frac{B_{3}^{T} A_{0}^{-1}}{m} & \frac{1}{m}
\end{array}\right),
$$

where

$$
m=1-B_{1}^{T} A_{0}^{-1} B_{1}-B_{2}^{T} A_{0}^{-1} B_{2}-B_{3}^{T} A_{0}^{-1} B_{3}
$$

and $I_{0}$ is the $(r-1) \times(r-1)$-dimensional identity matrix.

Step 5: the limit of $f_{1}\left(y_{1}, y_{2}\right)$.

Combining (7.78) and (7.74), one finds

$$
\begin{array}{r}
\mathbf{b}^{T} A^{-1} \mathbf{b}=\frac{r}{c} B^{T} A_{0}^{-1} B\left(\left(1-H_{1}\right) y_{1}^{2}+\left(1-H_{2}\right) y_{2}^{2}\right. \\
\left.+\frac{\left(\sqrt{H_{1}\left(1-H_{1}\right)} y_{1}+\sqrt{H_{2}\left(1-H_{2}\right)} y_{2}\right)^{2}}{H_{3}}\right)
\end{array}
$$




$$
=\left(\frac{r}{c}-1\right) \frac{y_{1}^{2}+y_{2}^{2}+2 y_{1} y_{2} \sqrt{\frac{H_{1} H_{2}}{\left(1-H_{1}\right)\left(1-H_{2}\right)}}}{\frac{H_{3}}{\left(1-H_{1}\right)\left(1-H_{2}\right)}}\left(1+o\left(\frac{1}{a}\right)\right) \text {. }
$$

Substituting the expression (7.79) and the formula (7.77) for the determinant into (7.73) yields

$$
f_{1}\left(y_{1}, y_{2}\right)=\frac{c}{r} \exp \left\{\left(\frac{r}{c}-1\right) \frac{y_{1}^{2}+y_{2}^{2}+2 y_{1} y_{2} \sqrt{\frac{H_{1} H_{2}}{\left(1-H_{1}\right)\left(1-H_{2}\right)}}}{2 \frac{H_{3}}{\left(1-H_{1}\right)\left(1-H_{2}\right)}}\right\}\left(1+o\left(\frac{1}{a}\right)\right) .
$$

Combining (7.80) with the asymptotic (7.69) of $\mathcal{J}_{1}$ and the relationship $f\left(y_{1}, y_{2}\right)=\mathcal{J}_{1} \times$ $f_{1}\left(y_{1}, y_{2}\right)$, we see $f\left(y_{1}, y_{2}\right)$ converges to the desired Gaussian density, which completes the proof of part (i) of the theorem when $P \sim \operatorname{GDP}(a, r, H)$.

The proof of part (ii) of this theorem follows from the same argument as that in the proof of part (ii) of Theorem 4.4.

\section{References}

Aomoto, K., Kita, M., Kohno, T., and Iohara, K. (2011). Theory of hypergeometric functions. Tokyo: Springer. 8

Bickel, P. J. and Wichura, M. J. (1971). "Convergence Criteria for Multiparameter Stochastic Processes and Some Applications." The Annals of Mathematical Statistics, 42(5): 1656-1670. 4, 27

Billingsley, P. (1995). Probability and measure. Wiley Series in Probability and Mathematical Statistics. John Wiley \& Sons, Inc., New York, third edition. A WileyInterscience Publication. 22, 27

Exton, H. (1976). Multiple hypergeometric functions and applications. Ellis Horwood: Chichester. 4, 28, 29

Favaro, S., Lijoi, A., Nava, C., Nipoti, B., Prünster, I., and Teh, Y. W. (2016). "On the Stick-Breaking Representation for Homogeneous NRMIs." Bayesian Analysis, 11(3): 697-724. 3, 4

Favaro, S., Lijoi, A., and Prünster, I. (2012). "On the stick-breaking representation of normalized inverse Gaussian priors." Biometrika, 99(3): 663-674. 2, 3

Gradshteyn, I. S. and Ryzhik, I. M. (2014). Table of integrals, series, and products. New York: Academic press, eight edition. 2, 10, 11, 12, 13

Hu, Y. (2017). Analysis on Gaussian spaces. World Scientific Publishing Co. Pte. Ltd., Hackensack, NJ. 20

$\mathrm{Hu}$, Y. and Zhang, J. (2020). "Dirichlet process and Bayesian nonparametric models (in Chinese)." Science in China, Chinese edition, To appear: 1-41. 2

Isserlis, L. (1918). "On a Formula for the Product-Moment Coefficient of any Order of 
a Normal Frequency Distribution in any Number of Variables." Biometrika, 12(1-2): 134-139. 27

Kim, N. and Bickel, P. J. (2003). "The limit distribution of a test statistic for bivariate normality." Statistica Sinica, 13: 327-349. 4

Lijoi, A., Mena, R. H., and Prünster, I. (2005a). "Bayesian Nonparametric Analysis for a Generalized Dirichlet Process Prior." Statistical Inference for Stochastic Processes, 8(3): 283-309. 28

- (2005b). "Hierarchical Mixture Modeling With Normalized Inverse-Gaussian Priors." Journal of the American Statistical Association, 100(472): 1278-1291. 4

Paisley, J. W., Zaas, A. K., Woods, C. W., Ginsburg, G. S., and Carin, L. (2010). "A stick-breaking construction of the beta process." In In International Conference on Machine Learning. Haifa, Israel. 4

Pitman, J. and Yor, M. (1997). "The two-parameter Poisson-Dirichlet distribution derived from a stable subordinator." The Annals of Probability, 25(2): 855-900. 2

Sethuraman, J. (1994). "A constructive definition of Dirichlet priors." Statistica sinica, 639-650. 1, 2

Straf, M. L. (1972). "Weak convergence of stochastic processes with several parameters." In Proceedings of the Sixth Berkeley Symposium on Mathematical Statistics and Probability, volume 2, 187-221. 5

Teh, Y. W., Grür, D., and Ghahramani, Z. (2007). "Stick-breaking construction for the Indian buffet process." In In Proceedings of the International Conference on Artificial Intelligence and Statistics, 11. San Juan, Puerto Rico. 4 